\title{
The induced semigroup of Schwarz maps to the space of Hilbert-Schmidt operators
}

\author{
George Androulakis · Alexander \\ Wiedemann · Matthew Ziemke
}

Received: date / Accepted: date

\begin{abstract}
We prove that for every semigroup of Schwarz maps on the von Neumann algebra of all bounded linear operators on a Hilbert space which has a subinvariant faithful normal state there exists an associated semigroup of contractions on the space of Hilbert-Schmidt operators of the Hilbert space. Moreover, we show that if the original semigroup is weak* continuous then the associated semigroup is strongly continuous. We introduce the notion of the extended generator of a semigroup on the bounded operators of a Hilbert space with respect to an orthonormal basis of the Hilbert space. We describe the form of the generator of a quantum Markov semigroup on the von Neumann algebra of all bounded linear operators on a Hilbert space which has an invariant faithful normal state under the assumption that the generator of the associated semigroup has compact resolvent.
\end{abstract}

Keywords Schwarz maps - strongly continuous semigroups · generator of a semigroup · form generator · faithful normal states $\cdot$ Moore-Penrose inverse

Mathematics Subject Classification (2010) 47D03 - 47D07 · 46L57 ·

81Q80

George Androulakis

Department of Mathematics, University of South Carolina, Columbia, SC

E-mail: giorgis@math.sc.edu

Alexander Wiedemann

Department of Mathematics, University of South Carolina, Columbia, SC

E-mail: alwiedemann@davidson.edu

Present address: Department of Mathematics and Computer Science, Davidson College, Davidson, NC

Matthew Ziemke

Department of Mathematics, Drexel University, Philadelphia, PA

E-mail: mjz55@drexel.edu 


\section{Introduction}

It is known that, under certain assumptions, semigroups on von Neumann algebras or their preduals give rise to associated semigroups on Hilbert spaces. Moreover, these associated semigroups often have stronger continuity properties than the original semigroups. For example, in [35, Equation (2.1)] it is stated that if $\left(T_{t}\right)_{t \geq 0}$ is a quantum Markov semigroup on a von Neumann algebra $\mathcal{A}$ which has an invariant faithful normal state, and if $(\mathcal{K}, \pi, \Omega)$ is the GNS triple associated to that state, then there exists a strongly continuous semigroup $\left(\overline{T_{t}}\right)_{t \geq 0}$ of contractions on $\mathcal{K}$ such that

$$
\overline{T_{t}}(\pi(A) \Omega)=\pi\left(T_{t}(A)\right) \Omega \text { for all } A \in \mathcal{A} \text { and } t \geq 0 \text {. }
$$

Since the proof of this statement is not included in 35] we provide a proof here (see Remarks 2 and 91). Other results which give rise to semigroups on Hilbert spaces starting from semigroups defined on spaces of operators can be found in literature. For example, in 34, Footnote of Theorem 6] it is proved that every strongly continuous semigroup $\left(T_{t}\right)_{t \geq 0}$ of positive isometries on the real Banach space of self-adjoint trace-class operators on a Hilbert space gives rise to a strongly continuous semigroup $\left(V_{t}\right)_{t \geq 0}$ of isometries on the Hilbert space such that $T_{t}$ is given as a conjugation by $V_{t}$ for all $t \geq 0$. In [19, Theorem 3] it is proved that, under appropriate assumptions, weakly continuous semigroups on $\mathcal{B}(\mathcal{H})$ (where $\mathcal{H}$ is a separable Hilbert space) give rise to corresponding semigroups of unitaries on some associated Hilbert space. In [31, Theorem 3.3.7] the author produces a strongly continuous group of unitaries associated with a norm continuous semigroup on the space of traceclass operators on a related Hilbert space.

In this work we prove a result similar to the result stated above in Equation (11) (Theorem 2). More precisely, we prove that every semigroup of Schwarz maps on $\mathcal{B}(\mathcal{H})$ (where $\mathcal{H}$ is a Hilbert space) which has an invariant faithful state gives rise to an associated semigroup $\left(\widetilde{T}_{t}\right)_{t \geq 0}$ of contractions on the space of Hilbert-Schmidt operators on $\mathcal{H}$. Our map is "more symmetric" than the one provided by Equation (1) (see the comments following Remark 2). We introduce the notion of the extended generator of a semigroup on bounded operators on a Hilbert space with respect to an orthonormal basis of the Hilbert space, and we explicitly describe how the generators of $\left(T_{t}\right)_{t \geq 0}$ and $\left(\widetilde{T}_{t}\right)_{t \geq 0}$ and the extended generator of $\left(T_{t}\right)_{t \geq 0}$ are related. We apply these descriptions to a quantum Markov semigroup $\left(T_{t}\right)_{t \geq 0}$ having an invariant faithful normal state under the assumption that the generator of $\left(\widetilde{T}_{t}\right)_{t \geq 0}$ has compact resolvent, which allows us to describe the form of the extended generator (and thereby the generator) of the semigroup $\left(T_{t}\right)_{t>0}$ with respect to an orthonormal basis, and thereby the generator itself (see 4). 


\subsection{Structure}

- In Section 2 we establish formal notation and definitions, and give some historical notes on the terminology.

- In Section 3 we consider several constructions arising from faithful, positive, normal functionals. In particular, in Subsection 3.1 we prove that every faithful positive normal functional on $\mathcal{B}(\mathcal{H})$ induces a canonical bounded linear map from $\mathcal{B}(\mathcal{H})$ to $\mathcal{S}_{2}(\mathcal{H})$. This map is used in Theorem 1 to prove that for every bounded linear Schwarz map on $\mathcal{B}(\mathcal{H})$, which has a subinvariant faithful positive functional, there exists a corresponding contraction on $\mathcal{S}_{2}(\mathcal{H})$. In Subsection 3.2 we consider an alternate construction for such induced maps using the GNS construction, and then compare and contrast the two methods.

- In Section 4 we recall the basic notions of continuity for semigroups, as well as formalize the definition of a semigroup's generator and its generator's domain. In Subsection 4.1 we introduce the notion of an extended generator, which can be defined on a larger domain while still agreeing with the usual generator on all finite subspaces. Theorem 2 relates the domains and actions of the generator, the extended generator, and the generator of the semigroup induced on $\mathcal{S}_{2}(\mathcal{H})$.

- In Section [5] we investigate the applications of Theorem 2 in the study of quantum Markov semigroups (QMSs), for which the exact form of the generator is known if the generator is bounded (see [28, and [36]). In Subsection 5.1. we describe the form of a QMS generator in the case that the generator of the semigroup induced on $\mathcal{S}_{2}(\mathcal{H})$ has compact resolvent.

\section{Preliminaries}

We first fix some notation. If $\mathcal{H}$ is a Hilbert space, let $\left(\mathcal{B}(\mathcal{H}),\|\cdot\|_{\infty}\right)$ denote the space of all bounded linear operators on $\mathcal{H}$. For $1 \leq p<\infty$, let $\left(\mathcal{S}_{p}(\mathcal{H}),\|\cdot\|_{p}\right)$ denote the Schatten- $p$ space of operators. In particular, $\left(\mathcal{S}_{2}(\mathcal{H}),\|\cdot\|_{2}\right)$ denotes the space of Hilbert-Schmidt operators on $\mathcal{H}$ and $\left(\mathcal{S}_{1}(\mathcal{H}),\|\cdot\|_{1}\right)$ denotes the space of trace-class operators on $\mathcal{H}$. Let $\langle\cdot, \cdot\rangle_{\mathcal{S}_{2}(\mathcal{H})}$ denote the inner product in $\mathcal{S}_{2}(\mathcal{H})$. If $L$ is a linear operator which is not necessarily bounded, then $D(L)$ will denote the domain of $L$.

We adopt the convention that functional will always mean bounded linear functional. Usually the functionals that we will consider will be faithful, positive, and normal, so this convention will help us to cut down the number of adjectives.

We would like to recall the Schwarz inequality and define the Schwarz maps. The classical Cauchy-Schwarz inequality states that $|\langle y, x\rangle| \leq\|y\|\|x\|$ for all vectors $x, y$ in a Hilbert space. This inequality is extended to $\left|\phi\left(y^{*} x\right)\right| \leq$ $\sqrt{\phi\left(y^{*} y\right)} \sqrt{\phi\left(x^{*} x\right)}$ for all $x, y$ in a $C^{*}$-algebra $\mathcal{A}$, where $\phi$ is a positive functional on $\mathcal{A}$ (see [33, Theorem 4.3.1]). The last inequality can be further extended to $\left(T\left(y^{*} x\right)\right)^{*} T\left(y^{*} x\right) \leq\left\|T\left(y^{*} y\right)\right\| T\left(x^{*} x\right)$ if $T$ is a completely positive map from a $C^{*}$-algebra $\mathcal{A}$ to the $C^{*}$-algebra $\mathcal{B}(\mathcal{H})$ of all bounded operators on a Hilbert 
space $\mathcal{H}$ (see [5, Lemma 2.6]). If in the last inequality one assumes that $\mathcal{A}$ is unital and $T$ is unital, then by replacing $y$ by the unit we obtain

$$
T(x)^{*} T(x) \leq T\left(x^{*} x\right) \quad \text { for all } x \in \mathcal{A} .
$$

A similar inequality was proved by Choi [11, Corollary 2.8] who proved that if $\mathcal{A}$ is a unital $C^{*}$-algebra and $T$ is a 2-positive unital map from $\mathcal{A}$ to $\mathcal{A}$ then $T\left(x^{*}\right) T(x) \leq T\left(x^{*} x\right)$ for all $x \in \mathcal{A}$. Choi calls the last inequality "Schwarz inequality". Similar inequalities appear in [32, Theorem 1] and [40, Theorem 7.4]. Since a positive linear map $T$ on a $C^{*}$-algebra $\mathcal{A}$ satisfies $T\left(x^{*}\right)=T(x)^{*}$ for all $x \in \mathcal{A}$, the last inequality is equivalent to (2). Following [41, page 14], we say that a bounded linear operator $T$ on a $C^{*}$-algebra $\mathcal{A}$ is a Schwarz map if Inequality (2) is satisfied. The advantage of Inequality (2), versus the inequality proved by Choi, is that Inequality (2) implies that $T$ is positive. Be warned that Inequality (2) is not homogeneous for $T$, and therefore by scaling the operator $T$ by a positive constant the above inequality is affected.

Next we recall the definition of invariant functionals and we define the notion of subinvariant positive functionals on a $C^{*}$-algebra. If $X$ is a Banach space, $T: X \rightarrow X$ is a bounded linear operator, and $\omega$ is a functional on $X$, then $\omega$ is called invariant for $\mathbf{T}$ if

$$
\omega(T x)=\omega(x) \text { for all } x \in X .
$$

If $\mathcal{A}$ is a $C^{*}$-algebra, $T: \mathcal{A} \rightarrow \mathcal{A}$ is a positive bounded linear operator, and $\omega$ is a positive functional on $\mathcal{A}$, then we will say that $\omega$ is subinvariant for $T$ if

$$
\omega(T a) \leq \omega(a) \text { for all } a \in \mathcal{A} \text { with } a \geq 0 .
$$

If $\mathcal{H}$ is a Hilbert space, then a functional $\omega$ on $\mathcal{B}(\mathcal{H})$ is called normal if and only if it is positive and continuous in the $\mathrm{w}^{*}$ topology. This is equivalent to the fact that there exists a unique positive operator $\rho \in \mathcal{S}_{1}(\mathcal{H})$ such that

$$
\omega(x)=\operatorname{Tr}(\rho x) \text { for all } x \in \mathcal{B}(\mathcal{H})
$$

where $\operatorname{Tr}$ denotes the trace. The positive functional $\omega$ associated to the positive trace-class operator $\rho$ via Equation (3) is denoted by $\omega_{\rho}$. If $\omega$ is a state (i.e. unital positive functional) on $\mathcal{B}(\mathcal{H})$ then $\omega$ is normal if and only if the positive trace-class operator $\rho$ which satisfies Equation (3) has trace equal to 1 . Note that if $\mathcal{H}$ is a Hilbert space and $T: \mathcal{B}(\mathcal{H}) \rightarrow \mathcal{B}(\mathcal{H})$ is a bounded linear operator, then a normal positive functional $\omega_{\rho}$ (for some positive trace-class operator $\rho$ ) is invariant for $T$ if and only if

$$
T^{\dagger}(\rho)=\rho,
$$

where $T^{\dagger}$ denotes the Banach dual operator of $T$ restricted to $\mathcal{S}_{1}(\mathcal{H})$ (viewed as a subspace of the dual of $\mathcal{B}(\mathcal{H})$ ). Also, if $\mathcal{H}$ is a Hilbert space and $T$ : $\mathcal{B}(\mathcal{H}) \rightarrow \mathcal{B}(\mathcal{H})$ is a positive bounded linear operator, then a normal positive functional $\omega_{\rho}$ (for some positive trace-class operator $\rho$ ) is subinvariant for $T$ if and only if

$$
T^{\dagger}(\rho) \leq \rho
$$


If $\mathcal{H}$ is a Hilbert space, recall that a positive functional $\omega$ on $\mathcal{B}(\mathcal{H})$ is faithful provided that $\omega(x)>0$ for all $x>0$. It is worth noting that $\mathcal{B}(\mathcal{H})$ has a faithful normal functional if and only if $\mathcal{H}$ is separable (see [6, Example $2.5 .5])$.

\section{Constructions Using Faithful, Positive, Normal Functionals}

We extensively use the next proposition, so we want to give it along with a proof.

Proposition 1 Let $\mathcal{H}$ be a Hilbert space and $\rho \in \mathcal{S}_{1}(\mathcal{H})$ be positive. Then the following are equivalent:

(i) the positive normal functional $\omega_{\rho}$ is faithful,

(ii) the operator $\rho$ is injective,

(iii) the operator $\rho$ has dense range.

Proof $[(i) \Rightarrow(i i)]$. Suppose $\omega_{\rho}$ is faithful. Let $h$ be a nonzero element of $\mathcal{H}$ and $P_{h}$ be the orthogonal projection onto the span of $h$. Then $P_{h}$ is a positive non-zero operator on $\mathcal{H}$. Hence, since $\omega_{\rho}$ is faithful,

$$
0<\omega_{\rho}\left(P_{h}\right)=\operatorname{Tr}\left(\rho P_{h}\right)=\operatorname{Tr}\left(\rho^{1 / 2} P_{h} P_{h} \rho^{1 / 2}\right)=\left\|P_{h} \rho^{1 / 2}\right\|_{2}^{2}=\frac{1}{\|h\|^{2}}\left\|\rho^{1 / 2} h\right\|^{2},
$$

and so $\rho^{1 / 2} h \neq 0$. By using the same argument with $h$ replaced by $\rho^{1 / 2} h$, we have that $\rho h \neq 0$. Thus, $\rho$ is injective.

$[(i) \Rightarrow($ iii $)]$. Assume that $\rho$ does not have dense range and let $P$ be the orthogonal projection to Range $(\rho)^{\perp}$. Then $P$ is a positive non-zero operator on $\mathcal{H}$, and so $\omega_{\rho}(P)>0$. However, $P \rho=0$, and so

$$
\omega_{\rho}(P)=\operatorname{Tr}(\rho P)=\operatorname{Tr}(P \rho)=\operatorname{Tr}(0)=0
$$

which is a contradiction. Thus, $\rho$ has dense range.

$[($ iii $) \Rightarrow(i)]$. Let $A \in \mathcal{B}(\mathcal{H})$ and suppose $\omega_{\rho}\left(A^{*} A\right)=0$. Then

$$
0=\omega_{\rho}\left(A^{*} A\right)=\operatorname{Tr}\left(\rho A^{*} A\right)=\operatorname{Tr}\left(\rho^{1 / 2} A^{*} A \rho^{1 / 2}\right)=\left\|A \rho^{1 / 2}\right\|_{2}^{2} .
$$

Hence $A \rho^{1 / 2}=0$, and therefore $A \rho=0$. Since $\rho$ has dense range, this implies $A=0$. Thus, $\omega_{\rho}$ is faithful.

$[(i i) \Rightarrow(i)]$. Assume that $\rho$ is injective and let $A \in \mathcal{B}(\mathcal{H})$ such that $\omega_{\rho}\left(A^{*} A\right)=0$. Equation (44) implies that $A \rho^{1 / 2}=0$ and hence $\rho^{1 / 2} A^{*}=0$, thus $\rho A^{*}=0$. This implies $\rho A^{*} x=0$ for any $x \in \mathcal{H}$, and since $\rho$ is injective we have that $A^{*} x=0$ for all $x \in \mathcal{H}$, and so $A=0$. Thus, $\rho$ is faithful.

Remark 1 Note that in the proof of $[(i) \Rightarrow(i i)]$ of the above proposition, we proved that (i) implies that $\rho^{1 / 2}$ is injective. Since $\rho^{1 / 2}=\rho^{1 / 4} \rho^{1 / 4}$ we immediately obtain that $\rho^{1 / 4}$ is injective. Since $\rho^{3 / 4}=\rho^{1 / 2} \rho^{1 / 4}$ we obtain that $\rho^{3 / 4}$ is injective as it is a composition of two injective maps. Further, since an operator is injective if and only if its adjoint has dense range, and $\rho^{1 / 4}, \rho^{1 / 2}$, and $\rho^{3 / 4}$ are self-adjoint, we have that $\rho^{1 / 4}, \rho^{1 / 2}$, and $\rho^{3 / 4}$ have dense range. 
3.1 Inducing Maps on $\boldsymbol{S}_{\mathbf{2}}(\boldsymbol{\mathcal { H }})$

Let $\mathcal{H}$ be a Hilbert space and fix $\rho \in \mathcal{S}_{1}(\mathcal{H})$ which is positive. Define

$$
i_{\rho}: \mathcal{B}(\mathcal{H}) \rightarrow \mathcal{B}(\mathcal{H}) \quad \text { by } \quad i_{\rho}(x)=\rho^{1 / 4} x \rho^{1 / 4} .
$$

The next proposition summarizes the properties of the map $i_{\rho}$. It is useful to first recall that for any Hilbert space $\mathcal{H}$ the following set inclusions hold:

$$
\mathcal{S}_{1}(\mathcal{H}) \subseteq \mathcal{S}_{2}(\mathcal{H}) \subseteq \mathcal{B}(\mathcal{H})
$$

Proposition 2 Let $\rho \in \mathcal{S}_{1}(\mathcal{H})$ be positive such that $\omega_{\rho}$ is a faithful positive functional. Then the following statements are valid:

(a) The map $i_{\rho}$ is injective.

(b) The map $i_{\rho}$ preserves positivity.

(c) The restriction $\left.i_{\rho}\right|_{\mathcal{S}_{2}(\mathcal{H})}$ of $i_{\rho}$ to $\mathcal{S}_{2}(\mathcal{H})$ is a contraction from $\mathcal{S}_{2}(\mathcal{H})$ into $\mathcal{S}_{1}(\mathcal{H})$.

(d) The map $i_{\rho}$ is a contraction from $\mathcal{B}(\mathcal{H})$ onto a dense subset of $\mathcal{S}_{2}(\mathcal{H})$.

Proof To prove $(\mathrm{a})$, let $x \in \mathcal{B}(\mathcal{H})$ and suppose $i_{\rho}(x)=0$. By Remark 1 we have that $\rho^{1 / 4}$ is injective. Therefore, since $\rho^{1 / 4} x \rho^{1 / 4}=0$, we obtain that $x \rho^{1 / 4}=0$. Further, since $\rho^{1 / 4}$ has dense range, (by Remark 1 again), we obtain that $x=0$. Thus $i_{\rho}$ is injective.

To prove $(\mathrm{b})$, let $x \in \mathcal{B}(\mathcal{H})$ where $x \geq 0$. Let $h \in \mathcal{H}$. Then

$$
\left\langle h, i_{\rho}(x) h\right\rangle=\left\langle h, \rho^{1 / 4} x \rho^{1 / 4} h\right\rangle=\left\langle\rho^{1 / 4} h, x \rho^{1 / 4} h\right\rangle \geq 0
$$

since $x \geq 0$. Thus $i_{\rho}$ maps positive operators to positive operators.

To prove (c), first note that for $p, q, r \geq 1$ with $\frac{1}{p}+\frac{1}{q}+\frac{1}{r}=1$ and for $x \in \mathcal{S}_{p}(\mathcal{H}), y \in \mathcal{S}_{q}(\mathcal{H})$, and $z \in \mathcal{S}_{r}(\mathcal{H})$, two applications of Holder's inequality give that $\|x y z\|_{1} \leq\|x\|_{p}\|y\|_{q}\|z\|_{r}$. From this we obtain that for $y \in \mathcal{S}_{2}(\mathcal{H})$ with $\|y\|_{2} \leq 1$ we have

$$
\left\|i_{\rho}(y)\right\|_{1}=\left\|\rho^{1 / 4} y \rho^{1 / 4}\right\|_{1} \leq\left\|\rho^{1 / 4}\right\|_{4}\|y\|_{2}\left\|\rho^{1 / 4}\right\|_{4}=\|\rho\|_{1}^{1 / 4}\|y\|_{2}\|\rho\|_{1}^{1 / 4} \leq\|\rho\|_{1}^{1 / 4} .
$$

To prove $(\mathrm{d})$, first notice that $i_{\rho}(x) \in \mathcal{S}_{2}(\mathcal{H})$ for all $x \in \mathcal{B}(\mathcal{H})$ since

$$
\begin{aligned}
\left\|i_{\rho}(x)\right\|_{2}^{2} & =\left\|\rho^{1 / 4} x \rho^{1 / 4}\right\|_{2}^{2}=\operatorname{Tr}\left(\rho^{1 / 2} x^{*} \rho^{1 / 2} x\right) \\
& \leq\left\|\rho^{1 / 2} x^{*} \rho^{1 / 2} x\right\|_{1} \leq\left\|\rho^{1 / 2} x^{*}\right\|_{2}\left\|\rho^{1 / 2} x\right\|_{2} \\
& =\operatorname{Tr}\left(\left(\rho^{1 / 2} x^{*}\right)^{*}\left(\rho^{1 / 2} x^{*}\right)\right)^{1 / 2} \operatorname{Tr}\left(\left(\rho^{1 / 2} x\right)^{*}\left(\rho^{1 / 2} x\right)\right)^{1 / 2} \\
& =\operatorname{Tr}\left(x \rho x^{*}\right)^{1 / 2} \operatorname{Tr}\left(x^{*} \rho x\right)^{1 / 2}<\infty .
\end{aligned}
$$

Let $y \in \mathcal{S}_{2}(\mathcal{H})$ such that $y \perp i_{\rho}(x)$ for all $x \in \mathcal{B}(\mathcal{H})$, (where the orthogonality is taken with respect to the Hilbert-Schmidt inner product). Then, for all $x \in \mathcal{B}(\mathcal{H})$, we have

$$
\begin{aligned}
0 & =\left\langle i_{\rho}(x), y\right\rangle_{\mathcal{S}_{2}(\mathcal{H})}=\left\langle\rho^{1 / 4} x \rho^{1 / 4}, y\right\rangle_{\mathcal{S}_{2}(\mathcal{H})} \\
& =\operatorname{Tr}\left(\rho^{1 / 4} x^{*} \rho^{1 / 4} y\right)=\left\langle x, \rho^{1 / 4} y \rho^{1 / 4}\right\rangle_{\mathcal{S}_{2}(\mathcal{H})}
\end{aligned}
$$


Therefore $\rho^{1 / 4} y \rho^{1 / 4}=0$. Since $\rho^{1 / 4}$ is injective, we have that $y \rho^{1 / 4}=0$ and, since $\rho^{1 / 4}$ has dense range, we have that $y=0$. Therefore $i_{\rho}$ has dense range.

To see that $\left\|i_{\rho}: \mathcal{B}(\mathcal{H}) \rightarrow \mathcal{S}_{2}(\mathcal{H})\right\| \leq 1$, let $x \in \mathcal{B}(\mathcal{H})$ and notice that

$$
\begin{aligned}
\left\|i_{\rho}(x)\right\|_{2} & =\sup _{\substack{y \in \mathcal{S}_{2}(\mathcal{H}) \\
\|y\|_{2} \leq 1}}\left|\left\langle i_{\rho}(x), y\right\rangle_{\mathcal{S}_{2}(\mathcal{H})}\right|=\sup _{\substack{y \in \mathcal{S}_{2}(\mathcal{H}) \\
\|y\|_{2} \leq 1}}\left|\operatorname{Tr}\left(i_{\rho}(x)^{*} y\right)\right| \\
& =\sup _{\substack{y \in \mathcal{S}_{2}(\mathcal{H}) \\
\|y\|_{2} \leq 1}}\left|\operatorname{Tr}\left(\rho^{1 / 4} y \rho^{1 / 4} x^{*}\right)\right| \leq \sup _{\substack{y \in \mathcal{S}_{2}(\mathcal{H}) \\
\|y\|_{2} \leq 1}}\left\|\rho^{1 / 4} y \rho^{1 / 4}\right\|_{1}\|x\|_{\infty} \\
& =\left\|\left.i_{\rho}\right|_{\mathcal{S}_{2}(\mathcal{H})}: \mathcal{S}_{2}(\mathcal{H}) \rightarrow \mathcal{S}_{1}(\mathcal{H})\right\|\|x\|_{\infty} \leq\|x\|_{\infty},
\end{aligned}
$$

where we used part (c) for the last inequality.

Definition 1 Let $\mathcal{H}$ be a Hilbert space and $\rho \in \mathcal{S}_{1}(\mathcal{H})$ be a positive operator. If $T: \mathcal{B}(\mathcal{H}) \rightarrow \mathcal{B}(\mathcal{H})$ is a bounded linear operator, we define the operator $\widetilde{T}: i_{\rho}(\mathcal{B}(\mathcal{H})) \rightarrow i_{\rho}(\mathcal{B}(\mathcal{H}))$ by

$$
\widetilde{T}\left(\rho^{1 / 4} x \rho^{1 / 4}\right)=\rho^{1 / 4} T(x) \rho^{1 / 4} \quad \text { for all } \quad x \in \mathcal{B}(\mathcal{H}) .
$$

Note that $\widetilde{T}$ depends on $\rho$ but, for simplicity, we chose notation which does not reflect this dependence.

The following theorem was first proven in [7. For the convenience of the reader we provide a proof of it here.

Theorem 1 Suppose $\mathcal{H}$ is a Hilbert space and $\rho \in \mathcal{S}_{1}(\mathcal{H})$ be a positive operator such that $\omega_{\rho}$ is a faithful positive functional on $\mathcal{B}(\mathcal{H})$. Let $T: \mathcal{B}(\mathcal{H}) \rightarrow \mathcal{B}(\mathcal{H})$ be a bounded linear operator which is a Schwarz map such that $\omega_{\rho}$ is a subinvariant functional for $T$. Then the corresponding operator $\widetilde{T}$ can be extended to all of $\mathcal{S}_{2}(\mathcal{H})$ as a contraction from $\mathcal{S}_{2}(\mathcal{H})$ to $\mathcal{S}_{2}(\mathcal{H})$.

Proof Since $\omega_{\rho}$ is a faithful normal functional on $\mathcal{B}(\mathcal{H})$, we have that $\mathcal{H}$ must be separable (see the comment above Proposition 1), so let $\left(e_{k}\right)_{k \geq 0}$ be an orthonormal basis for $\mathcal{H}$ which diagonalizes $\rho$ and let $P_{n}=\sum_{k=0}^{n}\left|e_{k}\right\rangle\left\langle e_{k}\right|$. Note that $\rho$ and its positive powers commute with each $P_{n}$. Define the linear subspace $\mathcal{A}=\left\{x \rho^{1 / 2}: x \in \mathcal{B}(\mathcal{H})\right\}$ and the map $\widehat{T}: \mathcal{A} \rightarrow \mathcal{A}$ by $\widehat{T}\left(x \rho^{1 / 2}\right)=$ $T(x) \rho^{1 / 2}$. Further, for $n \in \mathbb{N}$, define the map $\Delta_{n}: \mathcal{S}_{2}(\mathcal{H}) \rightarrow \mathcal{S}_{2}(\mathcal{H})$ by

$$
\Delta_{n}(x)=P_{n} \rho^{1 / 2} x \rho^{-1 / 2} P_{n} \quad \text { for all } \quad x \in \mathcal{S}_{2}(\mathcal{H})
$$


(note that $\rho^{1 / 2}$ is not invertible but $\rho^{-1 / 2} P_{n}$ is a bounded operator). Then, for any $x \in \mathcal{B}(\mathcal{H})$, we have

$$
\begin{aligned}
\left\|\widetilde{T}\left(i_{\rho}(x)\right)\right\|_{2}^{2} & =\left\|\rho^{1 / 4} T(x) \rho^{1 / 4}\right\|_{2}^{2}=\operatorname{Tr}\left(\rho^{1 / 4} T(x)^{*} \rho^{1 / 2} T(x) \rho^{1 / 4}\right) \\
& =\lim _{n \rightarrow \infty} \operatorname{Tr}\left(\rho^{1 / 2} T(x)^{*} P_{n} \rho^{1 / 2} T(x) \rho^{1 / 2} \rho^{-1 / 2} P_{n}\right) \\
& =\lim _{n \rightarrow \infty}\left\langle T(x) \rho^{1 / 2}, \Delta_{n}\left(T(x) \rho^{1 / 2}\right)\right\rangle_{\mathcal{S}_{2}(\mathcal{H})} \\
& =\lim _{n \rightarrow \infty}\left\langle\widehat{T}\left(x \rho^{1 / 2}\right), \Delta_{n} \widehat{T}\left(x \rho^{1 / 2}\right)\right\rangle_{\mathcal{S}_{2}(\mathcal{H})} \\
& =\lim _{n \rightarrow \infty}\left\langle x \rho^{1 / 2}, \widehat{T}^{*} \Delta_{n} \widehat{T}\left(x \rho^{1 / 2}\right)\right\rangle_{\mathcal{S}_{2}(\mathcal{H})}
\end{aligned}
$$

where we will see later on why $\widehat{T}^{*}$ is well-defined.

Define $\Delta: \mathcal{A} \rightarrow \mathcal{A}$ by $\Delta\left(x \rho^{1 / 2}\right)=\rho^{1 / 2} x$, which is well-defined since $\rho^{1 / 2}$ has dense range (hence, for $x, y \in \mathcal{B}(\mathcal{H}), x \rho^{1 / 2}=y \rho^{1 / 2}$ implies $x=y$ ). Let $\mathcal{B}=\{x \rho: x \in \mathcal{B}(\mathcal{H})\}$. We make the following three claims:

(i) $\widehat{T}$ is a contraction on $\mathcal{A}$. Therefore $\widehat{T}$ can be extended to a contraction on $\mathcal{S}_{2}(\mathcal{H})$ since $\mathcal{A}$ is dense in $\mathcal{S}_{2}(\mathcal{H})$ ).

(ii) $\Delta_{n}^{2}$ is positive. Therefore, by [37, Lemma 1.2], we have

$$
\widehat{T}^{*} \Delta_{n} \widehat{T} \leq\left(\widehat{T}^{*} \Delta_{n}^{2} \widehat{T}\right)^{1 / 2} .
$$

(iii) $\widehat{T}^{*} \Delta_{n}^{2} \widehat{T} \leq \Delta^{2}$ on $\mathcal{B}$. Thus,

$$
\left(\widehat{T}^{*} \Delta_{n}^{2} \widehat{T}\right)^{1 / 2} \leq\left(\Delta^{2}\right)^{1 / 2}=\Delta
$$

Hence, by combining (6) and (7), we obtain $\widehat{T}^{*} \Delta_{n} \widehat{T} \leq \Delta$ on $\mathcal{B}$.

Assume for the moment that the above claims (i), (ii), and (iii) are true. By replacing $x$ by $x \rho^{1 / 2}$, in Equation (5) we obtain that

$$
\begin{aligned}
\left\|\widetilde{T}\left(i_{\rho}\left(x \rho^{1 / 2}\right)\right)\right\|_{2}^{2} & =\lim _{n \rightarrow \infty}\left\langle x \rho, \widehat{T}^{*} \Delta_{n} \widehat{T}(x \rho)\right\rangle_{\mathcal{S}_{2}(\mathcal{H})} \\
& \leq\langle x \rho, \Delta(x \rho)\rangle_{\mathcal{S}_{2}(\mathcal{H})}=\left\langle x \rho, \rho^{1 / 2} x \rho^{1 / 2}\right\rangle_{\mathcal{S}_{2}(\mathcal{H})} \\
& =\operatorname{Tr}\left(\rho x^{*} \rho^{1 / 2} x \rho^{1 / 2}\right)=\operatorname{Tr}\left(\rho^{3 / 4} x^{*} \rho^{1 / 4} \rho^{1 / 4} x \rho^{3 / 4}\right) \\
& =\left\langle\rho^{1 / 4} x \rho^{3 / 4}, \rho^{1 / 4} x \rho^{3 / 4}\right\rangle_{\mathcal{S}_{2}(\mathcal{H})}=\left\|i_{\rho}\left(x \rho^{1 / 2}\right)\right\|_{2}^{2}
\end{aligned}
$$

and so $\widetilde{T}$ is a contraction on $i_{\rho}\left(\mathcal{B}(\mathcal{H}) \rho^{1 / 2}\right)$. We now show that $i_{\rho}\left(\mathcal{B}(\mathcal{H}) \rho^{1 / 2}\right)$ is dense in $\mathcal{S}_{2}(\mathcal{H})$. Let $y \in \mathcal{S}_{2}(\mathcal{H})$ such that $y \perp i_{\rho}\left(\mathcal{B}(\mathcal{H}) \rho^{1 / 2}\right)$. Then, for any $x \in \mathcal{B}(\mathcal{H})$ we have that

$$
\begin{aligned}
0 & =\left\langle i_{\rho}\left(x \rho^{1 / 2}\right), y\right\rangle_{\mathcal{S}_{2}(\mathcal{H})}=\operatorname{Tr}\left(i_{\rho}\left(x \rho^{1 / 2}\right)^{*} y\right) \\
& =\operatorname{Tr}\left(\rho^{1 / 4} \rho^{1 / 2} x^{*} \rho^{1 / 4} y\right)=\left\langle x, \rho^{1 / 4} y \rho^{3 / 4}\right\rangle_{\mathcal{S}_{2}(\mathcal{H})}
\end{aligned}
$$


and hence $\rho^{1 / 4} y \rho^{3 / 4}=0$. Since $\rho^{1 / 4}$ is injective, we then have that $y \rho^{3 / 4}=0$ and, since $\rho^{3 / 4}$ has dense range, we obtain that $y=0$. Therefore $i_{\rho}\left(\mathcal{B}(\mathcal{H}) \rho^{1 / 2}\right)$ is dense in $\mathcal{S}_{2}(\mathcal{H})$. Since $\widetilde{T}$ is a contraction on $i_{\rho}\left(\mathcal{B}(\mathcal{H}) \rho^{1 / 2}\right)$, we can extend it to a contraction on $\mathcal{S}_{2}(\mathcal{H})$. This finishes the proof of the theorem pending verification of claims (i), (ii), and (iii), as well as the fact that $\widehat{T}^{*}$ is welldefined. Then

First, we prove claim (i), i.e., that $\widehat{T}$ is a contraction on $\mathcal{A}$. Let $x \in \mathcal{B}(\mathcal{H})$.

$$
\begin{aligned}
\left\|\widehat{T}\left(x \rho^{1 / 2}\right)\right\|_{2}^{2} & =\left\|T(x) \rho^{1 / 2}\right\|_{2}^{2}=\left\langle T(x) \rho^{1 / 2}, T(x) \rho^{1 / 2}\right\rangle_{\mathcal{S}_{2}(\mathcal{H})} \\
& =\operatorname{Tr}\left(\rho^{1 / 2} T(x)^{*} T(x) \rho^{1 / 2}\right) \leq \operatorname{Tr}\left(\rho^{1 / 2} T\left(x^{*} x\right) \rho^{1 / 2}\right)
\end{aligned}
$$

since $T$ is a Schwarz map. Further,

$$
\begin{aligned}
\operatorname{Tr}\left(\rho^{1 / 2} T\left(x^{*} x\right) \rho^{1 / 2}\right) & =\operatorname{Tr}\left(\rho T\left(x^{*} x\right)\right) \leq \operatorname{Tr}\left(\rho x^{*} x\right)=\operatorname{Tr}\left(\rho^{1 / 2} x^{*} x \rho^{1 / 2}\right) \\
& =\left\langle x \rho^{1 / 2}, x \rho^{1 / 2}\right\rangle_{\mathcal{S}_{2}(\mathcal{H})}=\left\|x \rho^{1 / 2}\right\|_{2}^{2} .
\end{aligned}
$$

Therefore $\left\|\widehat{T}\left(x \rho^{1 / 2}\right)\right\|_{2}^{2} \leq\left\|x \rho^{1 / 2}\right\|_{2}^{2}$, and so $\widehat{T}$ is a contraction on $\mathcal{A}$. Hence, $\widehat{T}$ can be extended to a contraction on $\mathcal{S}_{2}(\mathcal{H})$ since $\mathcal{A}$ is dense in $\mathcal{S}_{2}(\mathcal{H})$ (this also shows that $\widehat{T}^{*}$ is well-defined).

For claim (ii), i.e., that $\Delta_{n}^{2}$ is positive, first note that since $\rho$ commutes with $P_{n}$ we have

$$
\Delta_{n}^{2} x=P_{n} \rho x P_{n} \rho^{-1} P_{n} \quad \text { for all } \quad x \in \mathcal{S}_{2}(\mathcal{H})
$$

(note that $\rho$ is not invertible but $\rho^{-1} P_{n}$ is a bounded operator). Indeed, if $x \in \mathcal{S}_{2}(\mathcal{H})$ then

$$
\begin{aligned}
\left\langle x, \Delta_{n}^{2} x\right\rangle_{\mathcal{S}_{2}(\mathcal{H})} & =\left\langle x, P_{n} \rho x P_{n} \rho^{-1} P_{n}\right\rangle_{\mathcal{S}_{2}(\mathcal{H})}=\operatorname{Tr}\left(x^{*} P_{n} \rho x P_{n} \rho^{-1} P_{n}\right) \\
& =\operatorname{Tr}\left(\rho^{1 / 2} P_{n} x P_{n} \rho^{-1 / 2} P_{n} P_{n} \rho^{-1 / 2} P_{n} x^{*} P_{n} \rho^{1 / 2}\right) \\
& =\operatorname{Tr}\left(\left(\rho^{1 / 2} P_{n} x P_{n} \rho^{-1 / 2} P_{n}\right)\left(\rho^{1 / 2} P_{n} x P_{n} \rho^{-1 / 2} P_{n}\right)^{*}\right) \geq 0,
\end{aligned}
$$

and so $\Delta_{n}^{2}$ is positive. By [37, Lemma 1.2], we then have that

$$
\widehat{T}^{*} \Delta_{n} \widehat{T} \leq\left(\widehat{T}^{*} \Delta_{n}^{2} \widehat{T}\right)^{1 / 2}
$$


It is left to prove claim (iii), i.e., that $\widehat{T}^{*} \Delta_{n}^{2} \widehat{T} \leq \Delta^{2}$ on $\mathcal{B}$. Indeed,

$$
\begin{aligned}
\left\langle x \rho, \widehat{T}^{*} \Delta_{n}^{2} \widehat{T}(x \rho)\right\rangle_{\mathcal{S}_{2}(\mathcal{H})} & =\left\langle T\left(x \rho^{1 / 2}\right) \rho^{1 / 2}, \Delta_{n}^{2} T\left(x \rho^{1 / 2}\right) \rho^{1 / 2}\right\rangle_{\mathcal{S}_{2}(\mathcal{H})} \\
& =\left\langle T\left(x \rho^{1 / 2}\right) \rho^{1 / 2}, P_{n} \rho T\left(x \rho^{1 / 2}\right) \rho^{1 / 2} P_{n} \rho^{-1} P_{n}\right\rangle_{\mathcal{S}_{2}(\mathcal{H})} \\
& =\operatorname{Tr}\left(\rho^{1 / 2} T\left(x \rho^{1 / 2}\right)^{*} P_{n} \rho T\left(x \rho^{1 / 2}\right) \rho^{1 / 2} P_{n} \rho^{-1} P_{n}\right) \\
& =\operatorname{Tr}\left(\rho T\left(x \rho^{1 / 2}\right) P_{n} T\left(x \rho^{1 / 2}\right)^{*} P_{n}\right) \\
& \leq \operatorname{Tr}\left(\rho T\left(x \rho^{1 / 2}\right) T\left(x \rho^{1 / 2}\right)^{*}\right) \quad(\text { see below }) \\
& \leq \operatorname{Tr}\left(\rho T\left(\left(x \rho^{1 / 2}\right)\left(x \rho^{1 / 2}\right)^{*}\right)\right) \quad(T \text { is a Schwarz map }) \\
& \leq \operatorname{Tr}\left(\rho\left(x \rho^{1 / 2}\right)\left(x \rho^{1 / 2}\right)^{*}\right) \quad\left(\omega_{\rho} \text { is subinvariant for } T\right) \\
& =\operatorname{Tr}\left(\rho x \rho x^{*}\right)=\operatorname{Tr}\left(\rho x(x \rho)^{*}\right)=\operatorname{Tr}\left((x \rho)^{*} \rho x\right) \\
& =\operatorname{Tr}\left((x \rho)^{*} \Delta^{2}(x \rho)\right) \quad\left(\text { since } \Delta^{2}(x \rho)=\rho x\right) \\
& =\left\langle x \rho, \Delta^{2}(x \rho)\right\rangle_{\mathcal{S}_{2}(\mathcal{H})} .
\end{aligned}
$$

This completes the proof as long as we justify the inequality (9). Indeed, we have that the inequality $\operatorname{Tr}\left(P_{n} A^{*} P_{n} A\right) \leq \operatorname{Tr}\left(A^{*} A\right)$ holds in general for any $A \in \mathcal{S}_{2}(\mathcal{H})$, since if $\left(e_{k}\right)_{k \geq 1}$ is the orthonormal basis of $\mathcal{H}$ used to define each $P_{n}$, then

$$
\begin{aligned}
\operatorname{Tr}\left(P_{n} A^{*} P_{n} A\right) & =\sum_{k=1}^{\infty}\left\langle e_{k}, P_{n} A^{*} P_{n}^{2} A e_{k}\right\rangle \\
& =\sum_{k=1}^{\infty}\left\langle P_{n} A P_{n} e_{k}, P_{n} A e_{k}\right\rangle=\sum_{k=1}^{n}\left\langle P_{n} A e_{k}, P_{n} A e_{k}\right\rangle
\end{aligned}
$$

and further

$$
\begin{aligned}
\sum_{k=1}^{n}\left\langle P_{n} A e_{k}, P_{n} A e_{k}\right\rangle & =\sum_{k=1}^{n}\left\|P_{n} A e_{k}\right\|^{2} \leq \sum_{k=1}^{n}\left\|P_{n}\right\|^{2}\left\|A e_{k}\right\|^{2} \\
& \leq \sum_{k=1}^{\infty}\left\|A e_{k}\right\|^{2}=\operatorname{Tr}\left(A^{*} A\right)
\end{aligned}
$$

\subsection{An Alternate Construction}

There is another situation where a bounded operator on a $C^{*}$-algebra gives rise to a corresponding operator on a Hilbert space, and we would like to mention this in the next remark. 
Remark 2 Let $\mathcal{A}$ be a unital $C^{*}$-algebra and $\omega$ be a faithful state on $\mathcal{A}$. Consider the GNS construction of $\mathcal{A}$ associated with $\omega$. Let $\mathcal{K}$ be the Hilbert space associated with the GNS construction, $\pi: \mathcal{A} \rightarrow \mathcal{B}(\mathcal{K})$ be the $*$-representation of $\mathcal{A}$ into the $C^{*}$-algebra of all bounded operators on $\mathcal{K}$, and $\Omega$ denote the cyclic element of the Hilbert space $\mathcal{K}$ for the representation $\pi$, (i.e. the subspace $\{\pi(a)(\Omega): a \in \mathcal{A}\}$ is norm dense in $\mathcal{K})$ which is equal to the unit of $\mathcal{A}$ viewed as an element of $\mathcal{K}$. Let $T$ be a bounded operator on $\mathcal{A}$ which is a Schwarz map. Assume that $\omega$ is subinvariant for $T$. Define an operator $\bar{T}$ on the dense subspace $\{\pi(a)(\Omega): a \in \mathcal{A}\}$ of $\mathcal{K}$ with values in $\mathcal{K}$ by

$$
\bar{T}(\pi(a)(\Omega))=\pi(T(a))(\Omega) \text { for all } a \in \mathcal{A} .
$$

Then $\bar{T}$ is a contraction (hence it extends to $\mathcal{K}$ ).

Proof Since $\omega$ is faithful, the quotient that is usually associated with the GNS construction does not take place, and the elements of $\mathcal{A}$ belong to $\mathcal{K}$. Let $\langle\cdot, \cdot\rangle_{\omega}$ denote the inner product in $\mathcal{K}$ and $\|\cdot\|_{\omega}$ denote the norm of $\mathcal{K}$. Then since $\omega$ is faithful, we have that for $a, b \in \mathcal{A},\langle a, b\rangle_{\omega}=\omega\left(a^{*} b\right)$ and hence $\|(\pi(a))(\Omega)\|_{\omega}^{2}=\omega\left(a^{*} a\right)$.

For every $a \in \mathcal{A}$ we have

$$
\begin{aligned}
\|\bar{T}(\pi(a)(\Omega))\|_{\omega}^{2}= & \|\pi(T(a))(\Omega)\|_{\omega}^{2}=\omega\left(T(a)^{*} T(a)\right) \leq \omega\left(T\left(a^{*} a\right)\right) \\
& (\text { since } \omega \text { is positive and } T \text { is a Schwarz map) } \\
\leq & \omega\left(a^{*} a\right) \quad(T \geq 0 \text { is a Schwarz map; } \omega \text { is subinvariant for } T) \\
= & \|\pi(a)(\Omega)\|_{\omega}^{2},
\end{aligned}
$$

which finishes the proof.

Notice the similarities between Theorem 1 and Remark 2 Both refer to a bounded operator on some $C^{*}$-algebra where a positive linear functional is fixed, and they each conclude the existence of an associated contraction on some Hilbert space. But there are three key differences between Theorem 1 and Remark 2, First, Theorem 1 refers to an operator on $\mathcal{B}(\mathcal{H})$ for some Hilbert space $\mathcal{H}$ (which is necessarily separable since $\mathcal{B}(\mathcal{H}$ ) is assumed to admit a faithful normal state), while Remark 2 assumes that the operator is defined on a general $C^{*}$-algebra. Second, the state $\omega_{\rho}$ which is mentioned in Theorem 1 is normal since it is defined via the trace-class operator $\rho$, while there is no such assumption in Remark 2 (the normality of the state $\omega$ in Remark 2 does not make sense in general since $\mathcal{A}$ is simply assumed to be a $C^{*}$-algebra and not a von Neumann algebra as it is assumed in [35. Equation (2.1)]). Third, the Hilbert space that is used in Theorem 1 is the space $\mathcal{S}_{2}(\mathcal{H})$ which does not depend on the positive linear functional, while the map $i_{\rho}$ which maps $\mathcal{B}(\mathcal{H})$ to $\mathcal{S}_{2}(\mathcal{H})$, does depend on the positive linear functional. On the other hand, the Hilbert space that is used in Remark 2 (i.e. the GNS construction associated to the faithful state $\omega$ of the $C^{*}$-algebra $\mathcal{A}$ ) depends on the state, while the $*$-representation $\pi$ of the von Neumann algebra which is associated with the GNS construction does not depend on the state. Notice also that 
the combinations of the Hilbert spaces with the representations in Theorem 1 and Remark 2 are very similar. More precisely, for $a, b \in \mathcal{B}(\mathcal{H})$ we have that $i_{\rho}(a), i_{\rho}(b) \in \mathcal{S}_{2}(\mathcal{H})$ hence

$$
\begin{aligned}
\left\langle i_{\rho}(a), i_{\rho}(b)\right\rangle_{\mathcal{S}_{2}(\mathcal{H})} & =\operatorname{Tr}\left(i_{\rho}(a)^{*} i_{\rho}(b)\right) \\
& =\operatorname{Tr}\left(\rho^{1 / 4} a^{*} \rho^{1 / 4} \rho^{1 / 4} b \rho^{1 / 4}\right)=\operatorname{Tr}\left(a^{*} \rho^{1 / 2} b \rho^{1 / 2}\right) .
\end{aligned}
$$

On the other hand, if we assume for the moment that the $C^{*}$-algebra $\mathcal{A}$ that appears in Remark 2 is equal to $\mathcal{B}(\mathcal{H})$ for some Hilbert space $\mathcal{H}$, and the faithful state $\omega$ on the $C^{*}$-algebra $\mathcal{A}$ is given by $\omega(a)=\operatorname{Tr}(\rho a)$ for some positive trace-class operator $\rho$ on $\mathcal{H}$, then the inner product of two elements $a, b \in \mathcal{A}$ via the GNS construction is given by

$$
\langle a, b\rangle_{\omega}=\omega\left(a^{*} b\right)=\operatorname{Tr}\left(\rho a^{*} b\right) .
$$

Thus the combination of the inner product with the representation that is used in Theorem 1 is slightly more "symmetric" than the combination of the inner product with the representation that is used in Remark2 The reader of course will notice the difference between the complexity of the proof of Theorem 1 and that of Remark 2. The extra intricacies in the proof of Theorem 1 is the price we pay in order to achieve the extra symmetry in the combination of the inner product and the representation as discussed above.

Remark 3 The assumption that " $\omega$ is subinvariant for $T$ " cannot be omitted in Remark 2 .

An example where $\omega$ is not a subinvariant functional for $T$ but all the other assumptions of Remark 2] are valid is presented in Remark 11.7 of [43].

Remark 4 Note that if $\mathcal{H}$ is a Hilbert space, $T: \mathcal{B}(\mathcal{H}) \rightarrow \mathcal{B}(\mathcal{H})$ is a bounded positive linear operator, and $\omega$ is a subinvariant positive faithful functional for $T$, then $\omega / \omega(1)$ is a subinvariant faithful state for $T$ (here 1 denotes the identity operator on $\mathcal{H}$ ). Thus, instead of assuming the existence of subinvariant positive faithful functionals, we henceforth simply assume the existence of subinvariant faithful states. Our subsequent results thus remain valid if the assumptions of the existence of subinvariant faithful states are replaced by the assumptions of the existence of subinvariant positive faithful functionals.

\section{Semigroups of Schwarz Maps}

We first recall some basic definitions about semigroups.

Definition 2 Let $X$ be a Banach space. A one-parameter family $\left(T_{t}\right)_{t \geq 0}$ of bounded operators on $X$ is a semigroup on $X$ if $T_{t+s}=T_{t} T_{s}$ for all $t, s \geq 0$, and $T_{0}=I$ where $I$ is the identity operator on $X$. We say the semigroup $\left(T_{t}\right)_{t \geq 0}$ on a Banach space $X$ is 
- uniformly continuous if the map $t \mapsto T_{t}$ is continuous with respect to the operator norm.

- strongly continuous if for all $x \in X$ the map $t \mapsto T_{t} x$ is continuous with respect to the norm on $X$.

- weakly continuous if for all $x \in X$ and all $x^{*} \in X^{*}$ the map $t \mapsto x^{*}\left(T_{t} x\right)$ is continuous.

- weak* continuous if $X$ is a dual Banach space $X=Y^{*}$ and for all $y \in Y$ and $x \in X$ the map $t \mapsto\left(T_{t}(x)\right)(y)$ is continuous.

If $\mathcal{H}$ is a Hilbert space and $X=\mathcal{B}(\mathcal{H})$ then the semigroup $\left(T_{t}\right)_{t \geq 0}$ on the Banach space $X$ is WOT continuous (where this acronym stands as usually for the weak operator topology) if for all $h_{1}, h_{2} \in \mathcal{H}$ and $x \in \mathcal{B}(\mathcal{H})$ we have that the map $t \mapsto\left\langle h_{1}, T_{t}(x) h_{2}\right\rangle$ is continuous.

It can be shown that a semigroup on a Banach space is strongly continuous if and only if it is weakly continuous (see [4, Thm. 3.31]). If $\left(T_{t}\right)_{t \geq 0}$ is a uniformly continuous semigroup on a Banach space $X$ then its generator is defined as the operator norm limit

$$
L=\lim _{t \rightarrow 0} \frac{T_{t}-I}{t}
$$

This limit exists and it defines a bounded operator on $X$. If we do not assume the uniform continuity of the semigroup, then the definition of the generator is given next:

Definition 3 Let $\left(T_{t}\right)_{t \geq 0}$ be a strongly continuous semigroup (resp. weakly continuous, resp. weak* continuous), on a Banach space $X$ (of course, when we assume that the semigroup is weak* continuous we assume that $X$ is a dual Banach space). We say an element $x \in X$ belongs to the domain $D(L)$ of the generator $L$ of $\left(T_{t}\right)_{t \geq 0}$, if

$$
\lim _{t \rightarrow 0} \frac{T_{t}(x)-x}{t}
$$

converges in norm (resp. weakly, resp. weak*) and, in this case, define the generator to be the generally unbounded operator $L$ such that

$$
L(x)=\lim _{t \rightarrow 0} \frac{T_{t}(x)-x}{t} \quad \text { for all } x \in D(L)
$$

where the last limit is taken in the norm (resp. weak, resp. weak*) topology of $X$.

Since a semigroup on a Banach space is strongly continuous if and only if it is weakly continuous, it is natural to ask whether the limits (10) and (11) can be replaced by weak limits and end up with the same $D(L)$ and $L$. It turns out that this is indeed the case (see [4, Proposition 3.36]). We will make use of this fact in the proof of Theorem 2 . 
4.1 The Extended Generator $\boldsymbol{L}_{\left(\boldsymbol{h}_{n}\right)}$ of $\left(\boldsymbol{T}_{\boldsymbol{t}}\right)_{\boldsymbol{t} \geq \mathbf{0}}$

We now wish to extend the definition of the generator to include some cases where the limit (11) does not exist. We first require the following notation:

Definition 4 Let $\mathcal{H}$ be a Hilbert space and $\left(h_{n}\right)_{n \in N}$ be an (countable or uncountable) orthonormal basis of $\mathcal{H}$. We let $M_{N}^{\left(h_{n}\right)}$ denote the set of all complex $N \times N$ matrices with rows and columns indexed by $N$. We view a matrix $L \in M_{N}^{\left(h_{n}\right)}$ as a linear map $L: D(L) \rightarrow \mathbb{C}^{N}$ acting on $\mathcal{H}$ as follows: denote $L=\left(L_{n, m}\right)_{n, m \in N}$, and define $D(L) \subset \mathcal{H}$ as the set of all vectors $h=\sum_{m \in N}\left\langle h_{m}, h\right\rangle h_{m} \in \mathcal{H}$ such that the series $\sum_{m \in N} L_{n, m}\left\langle h_{m}, h\right\rangle$ converges for all $n \in N$. Then

$$
L(h)=\left(\sum_{m \in N} L_{n, m}\left\langle h_{m}, h\right\rangle\right)_{n \in N} .
$$

This is in particular the natural matrix multiplication of $L$ against $h$ written as a column vector.

The following definition is given as the minimal requirements for the outputs of $L$ to be considered as linear maps in the sense given above for a fixed orthonormal basis $\left(h_{n}\right)_{n \in N}$ of $\mathcal{H}$.

Definition 5 Let $\mathcal{H}$ be a Hilbert space and $\left(h_{n}\right)_{n \in N}$ be a (countable or uncountable) orthonormal basis of $\mathcal{H}$. Let $\left(T_{t}\right)_{t \geq 0}$ be a semigroup of bounded operators on $\mathcal{B}(\mathcal{H})$. To define the extended generator $L_{\left(h_{n}\right)}$ of $\left(T_{t}\right)_{t>0}$ with respect to the basis $\left(h_{n}\right)_{n \in N}$ we first define its domain as the linear subspace of all $x \in \mathcal{B}(\mathcal{H})$ such that the function

$$
[0, \infty) \ni t \mapsto\left\langle h_{n}, T_{t}(x) h_{m}\right\rangle
$$

is differentiable at 0 for every $n, m \in N$; that is, $D\left(L_{\left(h_{n}\right)}\right)$ is the linear subspace of all $x \in \mathcal{B}(\mathcal{H})$ such that the limit

$$
\lim _{t \rightarrow 0}\left\langle h_{n}, \frac{T_{t}(x)-x}{t} h_{m}\right\rangle
$$

exists for every $n, m \in N$. In general $D\left(L_{\left(h_{n}\right)}\right)$ can be the zero subspace, but if the semigroup is WOT continuous then $D\left(L_{\left(h_{n}\right)}\right)$ is WOT dense in $\mathcal{B}(\mathcal{H})$. Define the extended generator $\mathbf{L}_{\left(\mathbf{h}_{\mathbf{n}}\right)}$ of $\left(\mathbf{T}_{\mathbf{t}}\right)_{\mathbf{t} \geq \mathbf{0}}$ (with respect to the orthonormal basis $\left.\left(\mathbf{h}_{\mathbf{n}}\right)_{\mathbf{n} \in \mathbf{N}}\right)$ to be the map with domain $D\left(L_{\left(h_{n}\right)}\right)$ whose range elements are matrices $L_{\left(h_{i}\right)}(x) \in M_{N}^{\left(h_{n}\right)}$ with entries given by

$$
\left[L_{\left(h_{i}\right)}(x)\right]_{n, m}=\lim _{t \rightarrow 0}\left\langle h_{n}, \frac{T_{t}(x)-x}{t} h_{m}\right\rangle .
$$


Next we want to compare the generator of a semigroup on $\mathcal{B}(\mathcal{H})$ with respect to an orthonormal basis of $\mathcal{H}$ to the usual generator. Since the definition of the generator depends on the continuity of the semigroup, in the next remark we will consider a weak* continuous semigroup on $\mathcal{B}(\mathcal{H})$ for some Hilbert space $\mathcal{H}$. The reason that we choose the weak* continuity versus any other continuity assumption is because it is the weakest and the most natural among all continuity assumptions that appear in Definition 2.

Remark 5 Let $\mathcal{H}$ be a Hilbert space, $\left(T_{t}\right)_{t \geq 0}$ be a weak* continuous semigroup of bounded operators on $\mathcal{B}(\mathcal{H})$, and let $L$ denote its generator. Let $\left(h_{n}\right)_{n \in N}$ be a (countable or uncountable) orthonormal basis of $\mathcal{H}$, and let $L_{\left(h_{n}\right)}$ denote the generator of $\left(T_{t}\right)_{t \geq 0}$ with respect to $\left(h_{n}\right)_{n \in N}$. Then $D(L) \subseteq D\left(L_{\left(h_{n}\right)}\right)$, and for every $x \in D(L)$ we have $L(x)=L_{\left(h_{n}\right)}(x)$, by which we mean the matrix of $L(x)$ with respect to $\left(h_{n}\right)_{n \in N}$ and the matrix $L_{\left(h_{n}\right)}(x)$ are equal.

Indeed, for fixed $x \in D(L)$ and every $h, h^{\prime} \in \mathcal{H}$ we have that

$$
\left\langle h, \frac{T_{t}(x)-x}{t} h^{\prime}\right\rangle \rightarrow\left\langle h, L(x) h^{\prime}\right\rangle \quad \text { as } t \rightarrow 0 .
$$

In particular,

$$
\lim _{t \rightarrow 0}\left\langle h_{n}, \frac{T_{t}(x)-x}{t} h_{m}\right\rangle=\left\langle h_{n}, L(x) h_{m}\right\rangle
$$

for every $n, m \in N$. Thus $x \in D\left(L_{\left(h_{n}\right)}\right)$.

Notation: If $N$ is a nonempty set, then we denote by $\Pi_{\mathrm{fin}}(N)$ the set of all finite subsets of $N$.

Notation: Let $\mathcal{H}$ and $\mathcal{K}$ be Hilbert spaces with $\mathcal{H} \subseteq \mathcal{K}$ and let $A \in \mathcal{B}(\mathcal{H})$ and $B \in \mathcal{B}(\mathcal{K})$. We shall denote by

$$
A=\operatorname{pr}_{\mathcal{H}}(B)
$$

the fact that

$$
A=\left.P_{\mathcal{H}} B\right|_{\mathcal{H}}
$$

where $\left.\right|_{\mathcal{H}}$ denotes the restriction to $\mathcal{H}$ and $P_{\mathcal{H}}: \mathcal{K} \rightarrow \mathcal{H}$ denotes the orthogonal projection from $\mathcal{K}$ onto $\mathcal{H}$. The operator $B$ is called a dilation of the operator $A$ and the operator $A$ is called a compression of the operator $B$.

Remark 6 Let $\mathcal{H}$ be a Hilbert space with (countable or uncountable) dimension $N,\left(h_{n}\right)_{n \in N}$ be an orthonormal basis of $\mathcal{H},\left(T_{t}\right)_{t \geq 0}$ be a semigroup of bounded operators on $\mathcal{B}(\mathcal{H})$, and let $L_{\left(h_{n}\right)}$ denote its generator with respect to $\left(h_{n}\right)_{n \in N}$. For $x \in D\left(L_{\left(h_{n}\right)}\right)$ and $F \in \Pi_{\text {fin }}(N)$ there exists a unique operator

$$
L_{\left(h_{n}\right)}(x)_{F}: \operatorname{Span}\left(h_{n}\right)_{n \in F} \rightarrow \operatorname{Span}\left(h_{n}\right)_{n \in F}
$$

satisfying

$$
\lim _{t \rightarrow 0}\left\langle h, \frac{T_{t}(x)-x}{t} h^{\prime}\right\rangle=\left\langle h, L_{\left(h_{n}\right)}(x)_{F} h^{\prime}\right\rangle \quad \text { for all } h, h^{\prime} \in \operatorname{Span}\left(h_{n}\right)_{n \in F},
$$


or equivalently

$$
\left\|\operatorname{pr}_{\operatorname{Span}\left(h_{n}\right)_{n \in F}}\left(\frac{T_{t}(x)-x}{t}\right)-L_{\left(h_{n}\right)}(x)_{F}\right\|_{\mathcal{B}\left(\operatorname{Span}\left(h_{n}\right)_{n \in F}\right)} \rightarrow 0 \quad \text { as } t \rightarrow 0 .
$$

Indeed, fix $F \in \Pi_{\text {fin }}(N)$. From Definition 5, $L_{\left(h_{n}\right)}(x)_{F}: \operatorname{Span}\left(x_{n}\right)_{n \in F} \rightarrow$ $\operatorname{Span}\left(x_{n}\right)_{n \in F}$ is uniquely defined by

$\left[L_{\left(h_{i}\right)}(x)_{F}\right](h)=\sum_{n, m \in F} \lim _{t \rightarrow 0}\left\langle h_{n}, \frac{T_{t}(x)-x}{t} h_{m}\right\rangle\left\langle h_{m}, h\right\rangle h_{n} \quad$ if $h=\sum_{m \in F}\left\langle h_{m}, h\right\rangle h_{m}$.

Then (13) is obvious from Definition 5 and (15). The equivalence of (13) and (14) then follows for any finite subset $F$ of $N$, since all linear Hausdorff topologies on the space of linear operators on $\operatorname{Span}\left(h_{n}\right)_{n \in F}$ are equivalent. Thus the WOT on $\operatorname{Span}\left(h_{n}\right)_{n \in F}$ in (13) can be replaced by the $\mathcal{B}\left(\operatorname{Span}\left(h_{n}\right)_{n \in F}\right)$ topology.

Remark 7 Let $\mathcal{H}$ be a Hilbert space with (countable or uncountable) dimension $N,\left(h_{n}\right)_{n \in N}$ be an orthonormal basis of $\mathcal{H},\left(T_{t}\right)_{t \geq 0}$ be a semigroup of bounded operators on $\mathcal{B}(\mathcal{H})$, and let $L_{\left(h_{n}\right)_{n}}$ denote its generator with respect to $\left(h_{n}\right)_{n \in N}$. Fix $x \in D\left(L_{\left(h_{n}\right)}\right)$. Then the family $\left(L_{\left(h_{n}\right)}(x)_{F}\right)_{F \in \Pi_{\mathrm{fin}}(N)}$ is compatible in the following sense: If $G \subset F$ are two finite subsets of $N$ then $\operatorname{pr}_{\operatorname{Span}\left(h_{n}\right)_{n \in G}}\left(L_{\left(h_{n}\right)}(x)_{F}\right)=L_{\left(h_{n}\right)}(x)_{G}$.

Indeed, this is obvious from (15).

Remark 8 The generator of a semigroup with respect to an orthonormal basis that we defined above is related to the form generator which was defined by Davies [13] and was further studied in [29, [9], 39, [30, [10, [20, 3], and 38. If $\left(T_{t}\right)_{t \geq 0}$ is a weak* continuous semigroup on $\mathcal{B}(\mathcal{H})$ for some Hilbert space $\mathcal{H}$, then a form generator is the map $\phi: \mathcal{K} \times \mathcal{B}(\mathcal{H}) \times \mathcal{K} \rightarrow \mathbb{C}$ where $\mathcal{K}$ is a dense linear subspace of $\mathcal{H}$, defined by

$$
\phi\left(h, x, h^{\prime}\right)=\left\langle h, \lim _{t \rightarrow 0} \frac{T_{t}(x)-x}{t} h^{\prime}\right\rangle \quad \text { for every } h, h^{\prime} \in \mathcal{K} \text { and every } x \in \mathcal{B}(\mathcal{H}) .
$$

Note that if $\left(h_{n}\right)_{n \in N}$ is an orthonormal basis of $\mathcal{H}$ and $\mathcal{K}$ denotes the linear span of $\left(h_{n}\right)_{n \in N}$ then the form generator coincides with the generator with respect to $\left(h_{n}\right)_{n \in N}$ if the domain of the generator with respect to $\left(h_{n}\right)_{n \in N}$ is equal to $\mathcal{B}(\mathcal{H})$. Here we assume that the domain of the generator with respect to an orthonormal basis is a linear subspace of $\mathcal{B}(\mathcal{H})$, not necessarily equal to $\mathcal{B}(\mathcal{H})$.

We require a few more definitions in order to state the next result.

Definition 6 Let $\mathcal{H}$ be a Hilbert space, $\omega$ be a state on $\mathcal{B}(\mathcal{H})$ and $\left(T_{t}\right)_{t \geq 0}$ be a semigroup of positive operators on $\mathcal{B}(\mathcal{H})$. We say that $\omega$ is a subinvariant state for the semigroup $\left(T_{t}\right)_{t \geq 0}$, if and only if $\omega$ is subinvariant for $T_{t}$ for every $t \geq 0$. 
Definition 7 The Moore-Penrose inverse or pseudoinverse $x^{(-1)}$ of $x \in$ $\mathcal{B}(\mathcal{H})$ is defined as the unique linear extension of $\left(\left.x\right|_{\mathcal{N}(x)^{\perp}}\right)^{-1}$, the inverse as a function, to

$$
D\left(x^{(-1)}\right):=\mathcal{R}(x)+\mathcal{R}(x)^{\perp}
$$

with $\mathcal{N}\left(x^{(-1)}\right)=\mathcal{R}(x)^{\perp}$, where $\mathcal{N}(x)$ and $\mathcal{R}(x)$ denote the nullspace and range of $x$, respectively. Letting $P$ and $Q$ denote the orthogonal projections onto $\mathcal{N}(x)$ and $\overline{\mathcal{R}(x)}$, respectively, it can be shown (see e.g. [17]) that $x^{(-1)}$ is uniquely determined by the relations

$$
x^{(-1)} x=I-P \quad \text { and } \quad x x^{(-1)}=\left.Q\right|_{D\left(x^{(-1)}\right)} .
$$

Notation: By $i_{\rho^{(-1)}}$ we mean the map from $\mathcal{B}(\mathcal{H})$ to the space of linear maps on $\mathcal{H}$ defined via

$$
i_{\rho^{(-1)}}(x)=\left(\rho^{1 / 4}\right)^{(-1)} x\left(\rho^{1 / 4}\right)^{(-1)} .
$$

Now we are ready to state the next result.

Theorem 2 Let $\mathcal{H}$ be a Hilbert space, $\left(T_{t}\right)_{t \geq 0}$ be a semigroup of Schwarz maps on $\mathcal{B}(\mathcal{H})$, and let $\rho \in \mathcal{S}_{1}(\mathcal{H})$ be such that $\omega_{\rho}$ is a faithful state on $\mathcal{B}(\mathcal{H})$ which is subinvariant for the semigroup $\left(T_{t}\right)_{t \geq 0}$. Then there exists a unique semigroup $\left(\widetilde{T}_{t}\right)_{t \geq 0}$ of contractions on $\mathcal{S}_{2}(\mathcal{H})$ such that

$$
\widetilde{T}_{t}\left(i_{\rho}(x)\right)=i_{\rho}\left(T_{t}(x)\right) \quad \text { for all } x \in \mathcal{B}(\mathcal{H})
$$

Moreover, if $\left(T_{t}\right)_{t \geq 0}$ is weak $k^{*}$-continuous then $\left(\widetilde{T}_{t}\right)_{t \geq 0}$ is strongly continuous. Let $L$ denote the generator of $\left(T_{t}\right)_{t \geq 0}$, let $\widetilde{L}$ denote the generator of $\left(\widetilde{T}_{t}\right)_{t \geq 0}$, and let $L_{\left(h_{n}\right)}$ denote the generator of $\left(T_{t}\right)_{t \geq 0}$ with respect to $\left(h_{n}\right)_{n \in \mathbb{N}}$, where $\left(h_{n}\right)_{n \in \mathbb{N}}$ is an orthonormal basis of $\mathcal{H}$ consisting of eigenvectors of $\rho$. Then $x \in D(L)$ implies $i_{\rho}(x) \in D(\widetilde{L})$, and moreover

$$
\widetilde{L}\left(i_{\rho}(x)\right)=i_{\rho}(L(x))
$$

conversely, $i_{\rho}(x) \in D(\widetilde{L})$ implies $x \in D\left(L_{\left(h_{n}\right)}\right)$, and moreover

$$
L_{\left(h_{n}\right)}(x)=i_{\rho^{(-1)}}\left(\widetilde{L}\left(i_{\rho}(x)\right)\right)
$$

Proof The operators $\widetilde{T}_{t}$ are well-defined by Theorem 11 Uniqueness comes from Equation (16) and the fact that $i_{\rho}(\mathcal{B}(\mathcal{H}))$ is dense in $\mathcal{S}_{2}(\mathcal{H})$. It is easy to see that $\widetilde{T}_{t+s}=\widetilde{T}_{t} \widetilde{T}_{s}$ and that $\widetilde{T}_{0}=1$ on $i_{\rho}(\mathcal{B}(\mathcal{H}))$, and the density of $i_{\rho}(\mathcal{B}(\mathcal{H}))$ implies these hold on all of $\mathcal{S}_{2}(\mathcal{H})$.

For the continuity statement, it suffices to assume that $\left(T_{t}\right)_{t \geq 0}$ is weak*continuous and show $\left(\widetilde{T}_{t}\right)_{t \geq 0}$ is strongly continuous on $i_{\rho}(\mathcal{B}(\mathcal{H}))$, since $i_{\rho}(\mathcal{B}(\mathcal{H}))$ 
is dense in $\mathcal{S}_{2}(\mathcal{H})$ and $\widetilde{T}_{t}$ is a contraction on $\mathcal{S}_{2}(\mathcal{H})$ for all $t \geq 0$. To this end, let $x \in \mathcal{B}(\mathcal{H})$. Then

$$
\begin{aligned}
\left\|\widetilde{T}_{t}\left(i_{\rho}(x)\right)-i_{\rho}(x)\right\|_{2}^{2}= & \left\|\rho^{1 / 4} T_{t}(x) \rho^{1 / 4}-\rho^{1 / 4} x \rho^{1 / 4}\right\|_{2}^{2} \\
= & \left\|\rho^{1 / 4} T_{t}(x) \rho^{1 / 4}\right\|_{2}^{2}+\left\|\rho^{1 / 4} x \rho^{1 / 4}\right\|_{2}^{2} \\
& -\left\langle\rho^{1 / 4} x \rho^{1 / 4}, \rho^{1 / 4} T_{t}(x) \rho^{1 / 4}\right\rangle_{\mathcal{S}_{2}(\mathcal{H})} \\
& -\left\langle\rho^{1 / 4} T_{t}(x) \rho^{1 / 4}, \rho^{1 / 4} x \rho^{1 / 4}\right\rangle_{\mathcal{S}_{2}(\mathcal{H})} \\
= & \left\|\widetilde{T}_{t}\left(i_{\rho}(x)\right)\right\|_{2}^{2}+\left\|i_{\rho}(x)\right\|_{2}^{2} \\
& -2 \Re\left\langle\rho^{1 / 4} x \rho^{1 / 4}, \rho^{1 / 4} T_{t}(x) \rho^{1 / 4}\right\rangle_{\mathcal{S}_{2}(\mathcal{H})} \\
\leq & 2\left\|i_{\rho}(x)\right\|_{2}^{2}-2 \Re\left(\operatorname{tr}\left(\rho^{1 / 4} x^{*} \rho^{1 / 4} \rho^{1 / 4} T_{t}(x) \rho^{1 / 4}\right)\right) \\
= & 2 \Re\left(\operatorname{tr}\left(\rho^{1 / 4} x^{*} \rho^{1 / 4} \rho^{1 / 4} x \rho^{1 / 4}-\rho^{1 / 4} x^{*} \rho^{1 / 4} \rho^{1 / 4} T_{t}(x) \rho^{1 / 4}\right)\right) \\
= & 2 \Re\left(\operatorname{tr}\left(\rho^{1 / 2} x^{*} \rho^{1 / 2}\left(x-T_{t}(x)\right)\right)\right) \rightarrow 0
\end{aligned}
$$

since $\rho^{1 / 2} x^{*} \rho^{1 / 2}$ is trace-class. Therefore $\left(\widetilde{T}_{t}\right)_{t \geq 0}$ is a strongly continuous semigroup of contractions on $\mathcal{S}_{2}(\mathcal{H})$.

To prove the final statement, first assume that $x \in D(L)$. Then

$$
\text { weak }^{*}-\lim _{t \rightarrow 0} \frac{T_{t}(x)-x}{t}=L(x) \text {. }
$$

Notice that for every $y \in \mathcal{S}_{2}(\mathcal{H})$ we obtain, by Proposition 2(c), that $\rho^{1 / 4} y^{*} \rho^{1 / 4} \in \mathcal{S}_{1}(\mathcal{H})$ and therefore the map $\mathcal{B}(\mathcal{H}) \ni z \mapsto \operatorname{Tr}\left(z \rho^{1 / 4} y^{*} \rho^{1 / 4}\right) \in \mathbb{C}$ is weak* continuous. Thus Equation (18) implies

$$
\operatorname{Tr}\left(\rho^{1 / 4} y^{*} \rho^{1 / 4} \frac{T_{t}(x)-x}{t}\right) \stackrel{t \rightarrow 0}{\longrightarrow} \operatorname{Tr}\left(\rho^{1 / 4} y^{*} \rho^{1 / 4} L(x)\right) ;
$$

that is,

$$
\left\langle y, \rho^{1 / 4} \frac{T_{t}(x)-x}{t} \rho^{1 / 4}\right\rangle_{\mathcal{S}_{2}(\mathcal{H})} \stackrel{t \rightarrow 0}{\longrightarrow}\left\langle y, \rho^{1 / 4} L(x) \rho^{1 / 4}\right\rangle_{\mathcal{S}_{2}(\mathcal{H})},
$$

and hence,

$$
\left\langle y, \frac{\widetilde{T}_{t}\left(\rho^{1 / 4} x \rho^{1 / 4}\right)-\rho^{1 / 4} x \rho^{1 / 4}}{t}\right\rangle_{\mathcal{S}_{2}(\mathcal{H})} \stackrel{t \rightarrow 0}{\longrightarrow}\left\langle y, \rho^{1 / 4} L(x) \rho^{1 / 4}\right\rangle_{\mathcal{S}_{2}(\mathcal{H})} .
$$

By [4, Proposition 3.36], we obtain that $\rho^{1 / 4} x \rho^{1 / 4} \in D(\widetilde{L})$ and $\widetilde{L}\left(\rho^{1 / 4} x \rho^{1 / 4}\right)=$ $\rho^{1 / 4} L(x) \rho^{1 / 4}$.

Conversely, by the Spectral Theorem there exists an orthonormal basis $\left(h_{n}\right)_{n \in \mathbb{N}}$ of $\mathcal{H}$ formed by eigenvectors of $\rho$. Let $L_{\left(h_{n}\right)}$ denote the generator of 
$\left(T_{t}\right)_{t \geq 0}$ with respect to $\left(h_{n}\right)_{n \in \mathbb{N}}$. Let $x \in \mathcal{B}(\mathcal{H})$ and assume that $\rho^{1 / 4} x \rho^{1 / 4} \in$ $D(\widetilde{L})$. Then we have that

$$
\frac{\widetilde{T}_{t}\left(\rho^{1 / 4} x \rho^{1 / 4}\right)-\rho^{1 / 4} x \rho^{1 / 4}}{t} \stackrel{t \rightarrow 0}{\longrightarrow} \widetilde{L}\left(\rho^{1 / 4} x \rho^{1 / 4}\right) \quad \text { in } \mathcal{S}_{2}(\mathcal{H})
$$

and hence

$$
\rho^{1 / 4} \frac{T_{t}(x)-x}{t} \rho^{1 / 4} \stackrel{t \rightarrow 0}{\longrightarrow} \widetilde{L}\left(\rho^{1 / 4} x \rho^{1 / 4}\right) \quad \text { in } \mathcal{S}_{2}(\mathcal{H})
$$

We will prove that $x \in D\left(L_{\left(h_{n}\right)}\right)$. Indeed, we have that

$$
\left\langle h, \rho^{1 / 4} \frac{T_{t}(x)-x}{t} \rho^{1 / 4} h^{\prime}\right\rangle \stackrel{t \rightarrow 0}{\longrightarrow}\left\langle h, \widetilde{L}\left(\rho^{1 / 4} x \rho^{1 / 4}\right) h^{\prime}\right\rangle
$$

for all $h, h^{\prime} \in \mathcal{H}$, so for any $n, m \in \mathbb{N}$ we may set $h=\left(\rho^{1 / 4}\right)^{(-1)} h_{n}$ and $h^{\prime}=\left(\rho^{1 / 4}\right)^{(-1)} h_{m}$ to obtain

$$
\begin{gathered}
\left\langle\left(\rho^{1 / 4}\right)^{(-1)} h_{n}, \rho^{1 / 4} \frac{T_{t}(x)-x}{t} \rho^{1 / 4}\left(\rho^{1 / 4}\right)^{(-1)} h_{m}\right\rangle \\
\stackrel{t \rightarrow 0}{\longrightarrow}\left\langle\left(\rho^{1 / 4}\right)^{(-1)} h_{n}, \widetilde{L}\left(\rho^{1 / 4} x \rho^{1 / 4}\right)\left(\rho^{1 / 4}\right)^{(-1)} h_{m}\right\rangle .
\end{gathered}
$$

Noting that $\left(\rho^{1 / 4}\right)^{*}=\rho^{1 / 4},\left(\left(\rho^{1 / 4}\right)^{(-1)}\right)^{*}=\left(\rho^{1 / 4}\right)^{(-1)}$, and $\rho^{1 / 4}\left(\rho^{1 / 4}\right)^{(-1)} h_{k}=$ $h_{k}$ for all $k \in \mathbb{N}$, this implies

$$
\left\langle h_{n}, \frac{T_{t}(x)-x}{t} h_{m}\right\rangle \stackrel{t \rightarrow 0}{\longrightarrow}\left\langle h_{n},\left(\rho^{1 / 4}\right)^{(-1)} \widetilde{L}\left(\rho^{1 / 4} x \rho^{1 / 4}\right)\left(\rho^{1 / 4}\right)^{(-1)} h_{m}\right\rangle .
$$

Because this limit exists for all $n, m \in \mathbb{N}$ we have $x \in D\left(L_{\left(h_{n}\right)}\right)$, and moreover

$$
L_{\left(h_{n}\right)}(x)=\left(\rho^{1 / 4}\right)^{(-1)} \widetilde{L}\left(\rho^{1 / 4} x \rho^{1 / 4}\right)\left(\rho^{1 / 4}\right)^{(-1)} .
$$

Remark 9 Since the proof of Equation (11) is not included in 35, we want to mention that its proof follows from our Remark 2 in a similar way that our Theorem 2 followed from our Theorem 1 (even the proof of the strong continuity of the semigroup $\left(\overline{T_{t}}\right)_{t \geq 0}$ follows the exact same argument as the proof of the strong continuity of the semigroup $\left(\widetilde{T}_{t}\right)_{t \geq 0}$ that appeared in Theorem 21). Moreover, the assumptions that the faithful state is normal and invariant for the semigroup and that the operators of the semigroup are completely positive that are mentioned in [35. for Equation (11) are not needed for its proof, because such assumptions were not used in Remark 2. Instead, for the validity of Equation (1), one merely needs to assume that the faithful state is subinvariant for the semigroup of Schwarz maps. Note also that, unlike Equation (1), Theorem 2 relates the generators of the two semigroups. 


\section{Applications to Quantum Markov Semigroups and Their Generators}

Since quantum Markov semigroups (QMSs) are semigroups of completely positive maps on von Neumann algebras (and hence 2-positive maps and hence Schwarz maps), we naturally obtain applications of Theorem 2 in the study of QMSs. The existence of invariant normal states for QMSs has been discussed in 22] and 24. Sufficient conditions for a semigroup to be decomposable into a sequence of irreducible semigroups each of them having an invariant normal state are given in 42] (see top half of page 608 , Theorem 5 on page 608 , and Proposition 5 on page 609). There are many results in the literature of semigroups which depend on the existence of invariant faithful normal states (for example, see [26, 27, 21, 25, and 8) and this assumption is often taken for granted as being physically reasonable. QMSs have been extensively studied since the 1970s with the exact form for the generators being one of the topics which has garnered a fair amount of attention. See for example [36, [28, [12, [14, 30, 1], 3], and 38. The generator of a QMS is a generally unbounded operator defined on a weak ${ }^{*}$ dense linear subspace of $\mathcal{B}(\mathcal{H})$. If the generator is bounded then the semigroup is uniformly continuous and the exact form of the generator was found in 28 and 36 . In this section, given a Hilbert space $\mathcal{H}$ and a QMS on $\mathcal{B}(\mathcal{H})$ having an invariant faithful normal state we study the associated semigroup of contractions on $\mathcal{S}_{2}(\mathcal{H})$. In particular, in Theorem 4 we describe the extended generator (and hence generator) of such a QMS under the assumption that the generator of the associated semigroup on $\mathcal{S}_{2}(\mathcal{H})$ has compact resolvent.

Definition 8 A quantum Markov semigroup (QMS) on $\mathcal{B}(\mathcal{H})$, (for some Hilbert space $\mathcal{H})$, is a weak ${ }^{*}$-continuous one-parameter semigroup of bounded linear operators acting on $\mathcal{B}(\mathcal{H})$, such that each member of the semigroup is completely positive and identity preserving.

Remark 10 If $\mathcal{H}$ is a Hilbert space and $\left(T_{t}\right)_{t \geq 0}$ is a QMS on $\mathcal{B}(\mathcal{H})$ which has a subinvariant normal state $\omega_{\rho}$ for some $\rho \in \mathcal{S}_{1}(\mathcal{H})$, then $\omega_{\rho}$ is in fact an invariant state for $\left(T_{t}\right)_{t \geq 0}$. Indeed for every $t \geq 0$,

$$
\operatorname{Tr}\left(T_{t}^{\dagger}(\rho)\right)=\operatorname{Tr}\left(T_{t}^{\dagger}(\rho) 1\right)=\operatorname{Tr}\left(\rho T_{t}(1)\right)=\operatorname{Tr}(\rho 1)=\operatorname{Tr}(\rho),
$$

which together with $T_{t}^{\dagger}(\rho) \leq \rho$ implies that $T_{t}^{\dagger}(\rho)=\rho$.

Usually the notion of complete positivity applies to maps on $C^{*}$-algebras. In particular, if the $C^{*}$-algebra is equal to $\mathcal{B}(\mathcal{H})$ for some Hilbert space $\mathcal{H}$, then the notion of complete positivity becomes equivalent to the following: A map $\mathcal{T}: \mathcal{B}(\mathcal{H}) \rightarrow \mathcal{B}(\mathcal{H})$ is completely positive if and only if for every $n \in \mathbb{N}$, $x_{1}, \ldots, x_{n} \in \mathcal{B}(\mathcal{H})$ and $h_{1}, \ldots, h_{n} \in \mathcal{H}$,

$$
\sum_{i, j=1}^{n}\left\langle h_{i}, \mathcal{T}\left(x_{i}^{*} x_{j}\right) h_{j}\right\rangle \geq 0 .
$$


Note that Equation (21) makes perfect sense even if the map $\mathcal{T}$ is not defined on a $C^{*}$-algebra, as long as $\mathcal{T}$ is defined on a Banach *-algebra $\mathcal{S}$ of operators on a Hilbert space $\mathcal{H}$. For example, $\mathcal{S}$ can be equal to $\mathcal{S}_{2}(\mathcal{H})$ and $\mathcal{T}$ can be a bounded linear operator from $\mathcal{S}$ to $\mathcal{S}$. We make this extension of the notion of complete positivity in the next definition.

Definition 9 Let $\mathcal{H}$ be a Hilbert space and $\mathcal{S}$ be a Banach *-algebra of bounded linear operators on $\mathcal{H}$. A bounded linear map $\mathcal{T}: \mathcal{S} \rightarrow \mathcal{S}$ will be called completely positive if for every $n \in \mathbb{N}, x_{1}, \ldots, x_{n} \in \mathcal{S}$ and $h_{1}, \ldots, h_{n} \in \mathcal{H}$, Equation (21) holds.

This terminology will be used in the next result.

Proposition 3 Let $\left(T_{t}\right)_{t \geq 0}$ be a weak ${ }^{*}$-continuous semigroup of Schwarz maps on $\mathcal{B}(\mathcal{H})$ for some Hilbert space $\mathcal{H}$ which possesses an invariant faithful normal state $\omega_{\rho}$ for some $\rho \in \mathcal{S}_{1}(\mathcal{H})$. Then the operators $T_{t}$ are completely positive for all $t \geq 0$ if and only if the operators $\widetilde{T}_{t}$ constructed in Theorem 2 are completely positive for all $t \geq 0$.

Proof First, assume $T_{t}$ is completely positive for $t \geq 0$, and let $x_{1}, x_{2}, \ldots, x_{n} \in$ $\mathcal{B}(\mathcal{H})$ and $h_{1}, h_{2}, \ldots, h_{n} \in \mathcal{H}$. Then

$$
\begin{aligned}
& \sum_{i, j=1}^{n}\left\langle h_{i}, \widetilde{T}_{t}\left(\left(\rho^{1 / 4} x_{i} \rho^{1 / 4}\right)^{*}\left(\rho^{1 / 4} x_{j} \rho^{1 / 4}\right)\right) h_{j}\right\rangle \\
= & \sum_{i, j=1}^{n}\left\langle\rho^{1 / 4} h_{i}, T_{t}\left(\left(\rho^{1 / 4} x_{i}\right)^{*}\left(\rho^{1 / 4} x_{j}\right)\right) \rho^{1 / 4} h_{j}\right\rangle \geq 0
\end{aligned}
$$

since $T_{t}$ is completely positive. Further, since the map $i_{\rho}$ from Proposition 2 has dense range, $\widetilde{T}_{t}$ is completely positive on $\mathcal{S}_{2}(\mathcal{H})$.

Conversely, assume $\widetilde{T}_{t}$ is completely positive for $t \geq 0$, and let $t \geq 0$, $x_{1}, x_{2}, \ldots, x_{n} \in \mathcal{B}(\mathcal{H})$ and $h_{1}, h_{2}, \ldots, h_{n} \in \mathcal{H}$. Then

$$
\sum_{i, j=1}^{n}\left\langle\rho^{1 / 4} h_{i}, T_{t}\left(x_{i}^{*} x_{j}\right) \rho^{1 / 4} h_{j}\right\rangle=\sum_{i, j=1}^{n}\left\langle h_{j}, \widetilde{T}_{t}\left(\left(x_{i} \rho^{1 / 4}\right)^{*}\left(x_{j} \rho^{1 / 4}\right)\right) h_{j}\right\rangle \geq 0
$$

since $\widetilde{T}_{t}$ is completely positive. Because the map $\rho^{1 / 4}$ has dense range, this is sufficient to show $T_{t}$ is completely positive.

For the next result, recall the notion of conditionally completely positive maps introduced by Lindblad in [36]. A linear operator $L: D(L)(\subseteq \mathcal{B}(\mathcal{H})) \rightarrow$ $\mathcal{B}(\mathcal{H})$ is called conditionally completely positive if for all $n \in \mathbb{N}$, for all $a_{1}, a_{2}, \ldots, a_{n} \in \mathcal{B}(\mathcal{H})$ such that $a_{i}^{*} a_{j} \in D(L)$ for all $i, j=1,2, \ldots, n$, that for all $h_{1}, h_{2}, \ldots h_{n} \in \mathcal{H}$ with $\sum_{i=1}^{n} a_{i}\left(h_{i}\right)=0$, we have that

$$
\sum_{i, j=1}^{n}\left\langle h_{i}, L\left(a_{i}^{*} a_{j}\right) h_{j}\right\rangle \geq 0
$$


The next result is known for uniformly continuous semigroups. For example, see [20, Proposition 3.12 and Lemma 3.13], or see [18, Proposition 2.9]. In fact the known proof works for a more general setting as the next result indicates.

Theorem 3 Let $\mathcal{S}$ be a Banach *-algebra of operators acting on a Hilbert space $\mathcal{H}$.

1. Let $\left(\mathcal{T}_{t}\right)_{t \geq 0}$ be a WOT continuous semigroup on $\mathcal{S}$ and let $\mathcal{L}$ be its generator. If $\mathcal{T}_{t}$ is completely positive for all $t \geq 0$ then $\mathcal{L}\left(a^{*}\right)=\mathcal{L}(a)^{*}$ for all $a \in D(\mathcal{L})$ and $\mathcal{L}$ is conditionally completely positive.

2. Let $\left(\mathcal{T}_{t}\right)_{t \geq 0}$ be a uniformly continuous semigroup on $\mathcal{S}$ with generator $\mathcal{L}$. If $\mathcal{L}\left(a^{*}\right)=\mathcal{L}(a)^{*}$ for all $a \in \mathcal{S}$ and $\mathcal{L}$ is conditionally completely positive, then $\mathcal{T}_{t}$ is completely positive for all $t \geq 0$.

Proof The proof of (2) immediately follows from [20, Proposition 3.12 and Lemma 3.13]. To prove (1), suppose $a_{1}, a_{2}, \ldots, a_{n} \in \mathcal{S}$ such that $a_{i}^{*} a_{j} \in D(L)$ for all $i, j=1, \ldots, n$ and $h_{1}, h_{2}, \ldots, h_{n} \in \mathcal{H}$ such that $\sum_{i=1}^{n} a_{i}\left(h_{i}\right)=0$. Then,

$$
\begin{aligned}
\sum_{i, j=1}^{n}\left\langle h_{i}, \mathcal{L}\left(a_{i}^{*} a_{j}\right) h_{j}\right\rangle & =\lim _{t \rightarrow 0^{+}} \sum_{i, j=1}^{n} \frac{1}{t}\left\langle h_{i},\left(T_{t}-1\right)\left(a_{i}^{*} a_{j}\right) h_{j}\right\rangle \\
& =\lim _{t \rightarrow 0^{+}} \sum_{i, j=1}^{n} \frac{1}{t}\left\langle h_{i}, T_{t}\left(a_{i}^{*} a_{j}\right) h_{j}\right\rangle \quad\left(\text { since } \sum_{i=1}^{n} a_{i}\left(h_{i}\right)=0\right) \\
& \geq 0
\end{aligned}
$$

since $T_{t}$ is completely positive for all $t \geq 0$.

Corollary 1 Let $\mathcal{H}$ be a Hilbert space and $\left(T_{t}\right)_{t \geq 0}$ be a $Q M S$ on $\mathcal{B}(\mathcal{H})$ which possesses an invariant faithful normal state $\omega_{\rho}$ for some $\rho \in \mathcal{S}_{1}(\mathcal{H})$. Let $\widetilde{L}$ be the generator of the strongly continuous semigroup $\left(\widetilde{T}_{t}\right)_{t \geq 0}$ of contractions on $\mathcal{S}_{2}(\mathcal{H})$ defined in Theorem 国. Then $\widetilde{L}\left(a^{*}\right)=\widetilde{L}(a)^{*}$ for all $a \in D(\widetilde{L})$ and $\widetilde{L}$ is conditionally completely positive.

Proof The proof follows immediately from Proposition 3 and Theorem 3(1).

5.1 The Form of $\boldsymbol{L}_{\left(\boldsymbol{h}_{n}\right)}$ when the Resolvent of $\widetilde{\boldsymbol{L}}$ is Compact

In this subsection we consider the form of the extended generator $L_{\left(h_{n}\right)}$ when the resolvent of $\widetilde{L}$ is compact, by which we mean that $(\widetilde{L}-\lambda)^{-1}$ is compact for some $\lambda$ in the resolvent set of $\widetilde{L}$ (equivalently all $\lambda$ in the resolvent set, by the resolvent identity). Notably, this assumption implies $\widetilde{L}$ is necessarily unbounded if $\mathcal{H}$ is infinite dimensional, since the composition of bounded with compact is compact but $I=(\widetilde{L}-\lambda)(\widetilde{L}-\lambda)^{-1}$ is not compact. More information about operators on a Hilbert space with compact resolvent can be found in Theorems XIII.4.1 and XIII.4.2 of [15]. Examples of such operators are the 
diagonal operator with eigenvalues $1,2,3, \ldots$, or the Laplacian on a bounded domain of $\mathbb{R}^{d}$.

We will make use of the following two notations:

Notation: Let $\mathcal{H}$ be a Hilbert space and $w, z \in \mathcal{S}_{2}(\mathcal{H})$. Define $M_{w} \otimes z$ : $\mathcal{S}_{2}(\mathcal{H}) \otimes \mathcal{H} \rightarrow \mathcal{S}_{2}(\mathcal{H}) \otimes \mathcal{H}$ by

$$
M_{w} \otimes z\left(\sum_{i=1}^{k} x_{i} \otimes h_{i}\right)=\sum_{i=1}^{k} x_{i} w \otimes z\left(h_{i}\right) .
$$

Notation: Let $\mathcal{H}$ be a Hilbert space and $e \in \mathcal{H}$ such that $\|e\|=1$. Define $T_{e}: \mathcal{S}_{2}(\mathcal{H}) \otimes \mathcal{H} \rightarrow \mathcal{S}_{2}(\mathcal{H}) \otimes \mathcal{H}$ by

$$
T_{e}\left(\sum_{i=1}^{k} x_{i} \otimes h_{i}\right)=\sum_{i=1}^{k}\left|x_{i}\left(h_{i}\right)\right\rangle\langle e| \otimes e .
$$

We are now ready to state the main result:

Theorem 4 Let $\mathcal{H}$ be a Hilbert space, $\left(T_{t}\right)_{t \geq 0}$ be a $Q M S$ on $\mathcal{B}(\mathcal{H})$ having an invariant faithful normal state $\omega_{\rho}$ for some $\rho \in \mathcal{S}_{1}(\mathcal{H})$, and $L$ be the generator of $\left(T_{t}\right)_{t \geq 0}$. Let $\left(\widetilde{T}_{t}\right)_{t \geq 0}$ be the strongly continuous semigroup of contractions on $\mathcal{S}_{2}(\mathcal{H})$ defined in Theorem 2 and let $\widetilde{L}$ be its generator. Assume that the generator $\widetilde{L}$ has compact resolvent. Then the following assertions are valid:

(a) There exist complete orthonormal families $\left(a_{n}\right)_{n \in \mathbb{N}}$ and $\left(b_{n}\right)_{n \in \mathbb{N}}$ of selfadjoint elements in $\mathcal{S}_{2}(\mathcal{H})$ and a sequence of positive scalars $\left(\lambda_{n}\right)_{n \in \mathbb{N}}$ with $\lambda_{n} \rightarrow \infty$ as $n \rightarrow \infty$ (if $\mathcal{H}$ is infinite dimensional) such that

$$
\widetilde{L}=I+\sum_{n=1}^{\infty} \lambda_{n}\left|a_{n}\right\rangle\left\langle b_{n}\right|
$$

where the sum converges in the SOT (if it is infinite), i.e. for every $x \in$ $D(\widetilde{L})$ we have that $\widetilde{L}(x)=x+\sum_{n=1}^{\infty} \lambda_{n}\left\langle b_{n}, x\right\rangle a_{n}$ with $\sum_{n}\left|\lambda_{n}\left\langle b_{n}, x\right\rangle\right|^{2}<\infty$.

(b) By the Spectral Theorem there is an orthonormal basis $\left(h_{n}\right)_{n \in \mathbb{N}}$ of $\mathcal{H}$ which consists of eigenvectors of $\rho$. Let $L_{\left(h_{n}\right)}$ denote the generator of $\left(T_{t}\right)_{t \geq 0}$ with respect to $\left(h_{n}\right)_{n \in \mathbb{N}}$. Then

$$
L_{\left(h_{n}\right)}=I+\sum_{n=1}^{\infty} \lambda_{n}\left|i_{\rho^{(-1)}}\left(a_{n}\right)\right\rangle\left\langle i_{\rho}\left(b_{n}\right)\right|
$$

where the sum converges in the SOT (if it is infinite). We note that $\left|i_{\rho^{(-1)}}\left(a_{n}\right)\right\rangle\left\langle i_{\rho}\left(b_{n}\right)\right|$ has domain $\mathcal{B}(\mathcal{H})$ for all $n$, since

$$
\left|i_{\rho^{(-1)}}\left(a_{n}\right)\right\rangle\left\langle i_{\rho}\left(b_{n}\right)\right| x=\left\langle i_{\rho}\left(b_{n}\right), x\right\rangle i_{\rho^{(-1)}}\left(a_{n}\right)=\left\langle b_{n}, i_{\rho}(x)\right\rangle i_{\rho^{(-1)}}\left(a_{n}\right)
$$

and $b_{n}, i_{\rho}(x) \in \mathcal{S}_{2}(\mathcal{H})$. Explicitly, for every $x \in D\left(L_{\left(h_{n}\right)}\right)$ and every $i, j \in \mathbb{N}$ we have that

$$
\left\langle h_{i},\left[L_{\left(h_{n}\right)}(x)\right] h_{j}\right\rangle=\left\langle h_{i}, x h_{j}\right\rangle+\sum_{n=1}^{\infty} \lambda_{n}\left\langle b_{n}, i_{\rho}(x)\right\rangle\left\langle h_{i}, i_{\rho^{(-1)}}\left(a_{n}\right) h_{j}\right\rangle .
$$


(c) We have

$$
I=-\sum_{n=1}^{\infty} \lambda_{n}\left\langle b_{n}, \rho^{1 / 2}\right\rangle i_{\rho^{(-1)}}\left(a_{n}\right),
$$

where the sum converges in the SOT (if it is infinite).

(d) For all $e \in \mathcal{H}$ with $\|e\|=1$ we have that the operator $\widetilde{L}_{\otimes, e}: \mathcal{S}_{2}(\mathcal{H}) \otimes \mathcal{H} \rightarrow$ $\mathcal{S}_{2}(\mathcal{H}) \otimes \mathcal{H}$ is positive, where the operator $\widetilde{L}_{\otimes, e}$ is defined by

$$
\widetilde{L}_{\otimes, e}=\left(I d+T_{e}^{*}\right)\left(\sum_{n=1}^{\infty} \lambda_{n} M_{b_{n}} \otimes a_{n}\right)\left(I d+T_{e}\right)
$$

where Id stands for the identity operator on $\mathcal{S}_{2}(\mathcal{H}) \otimes \mathcal{H}$ and the sum converges in the SOT (if it is infinite).

We note that the sum in (22) is finite if and only if $\mathcal{H}$ is finite dimensional. Indeed, if $\widetilde{L}$ is bounded with compact resolvent then $\mathcal{H}$ is finite dimensional, as remarked in the preamble of this subsection. The proof of Theorem 4 is at the end of this subsection, after the following three results:

Lemma 1 Let $\mathcal{H}$ be a separable Hilbert space and $A$ be an invertible linear operator on $S_{2}(\mathcal{H})$ with dense domain which is closed under adjoints. If $A$ satisfies $A\left(a^{*}\right)=(A(a))^{*}$ for all $a \in D(A)$, then $D\left(A^{\dagger}\right)$ and $D\left(A^{-1}\right)$ are closed under adjoints, $A^{\dagger}\left(b^{*}\right)=\left(A^{\dagger}(b)\right)^{*}$ for all $b \in D\left(A^{\dagger}\right)$, and $A^{-1}\left(c^{*}\right)=\left(A^{-1}(c)\right)^{*}$ for all $c \in D\left(A^{-1}\right)$.

Proof Let $a \in D(A)$ and $b \in D\left(A^{\dagger}\right)$. Then

$$
\begin{aligned}
\left|\left\langle A(a), b^{*}\right\rangle\right| & =\left|\left\langle\left(A\left(a^{*}\right)\right)^{*}, b^{*}\right\rangle\right|=\left|\left\langle b, A\left(a^{*}\right)\right\rangle\right| \\
& =\left|\left\langle A^{\dagger}(b), a^{*}\right\rangle\right|=\left|\left\langle a,\left(A^{\dagger}(b)\right)^{*}\right\rangle\right| \leq\left\|a \left|\left\|||\left(A^{\dagger}(b)\right)^{*}\right\|,\right.\right.
\end{aligned}
$$

and so $b^{*} \in D\left(A^{\dagger}\right)$ by definition. As before,

$$
\left\langle a, A^{\dagger}\left(b^{*}\right)\right\rangle=\left\langle A(a), b^{*}\right\rangle=\left\langle a,\left(A^{\dagger}(b)\right)^{*}\right\rangle
$$

and since $D(A)$ is dense this implies $A^{\dagger}\left(b^{*}\right)=\left(A^{\dagger}(b)\right)^{*}$ for all $b \in D\left(A^{\dagger}\right)$. Further, for every $c \in D\left(A^{-1}\right)$ there exists an $a \in D(A)$ such that $A(a)=$ $c$. Since $A$ is star-preserving we have that $A\left(a^{*}\right)=c^{*}$. Then, by definition, $\left(A^{-1}(c)\right)^{*}=a^{*}=A^{-1}\left(c^{*}\right)$.

Lemma 2 Let $\mathcal{H}$ be a Hilbert space and $A$ be a compact and self-adjoint linear operator on $S_{2}(\mathcal{H})$. Then $A$ satisfies $A\left(a^{*}\right)=(A(a))^{*}$ for all $a \in S_{2}(\mathcal{H})$ if and only if

$$
A=\sum_{n=1}^{\infty} \lambda_{n}\left|x_{n}\right\rangle\left\langle x_{n}\right|
$$

with $\left(\lambda_{n}\right)_{n=1}^{\infty} \subseteq \mathbb{R}$ and $\left(x_{n}\right)_{n=1}^{\infty}$ an orthonormal basis of $S_{2}(\mathcal{H})$ consisting of self-adjoint operators. 
Proof If $A$ is compact and self-adjoint, then the Spectral Theorem implies there is an eigensystem decomposition

$$
A=\sum_{n=1}^{\infty} \lambda_{n}\left|y_{n}\right\rangle\left\langle y_{n}\right|,
$$

with $\left(\lambda_{n}\right)_{n=1}^{\infty} \subseteq \mathbb{R}$ and $\left(y_{n}\right)_{n=1}^{\infty}$ an orthonormal basis of $S_{2}(\mathcal{H})$. Because $A$ is self-adjoint and star-preserving, we have that $A\left(y_{n}\right)=\lambda_{n} y_{n}$ implies $A\left(y_{n}^{*}\right)=\lambda_{n} y_{n}^{*}$. Thus, every eigenspace of $A$ is self-adjoint. Let $E$ be an eigenspace of $A$ corresponding to a fixed eigenvalues, and consider the orthonormal basis $\left(y_{n_{j}}\right)_{j=1}^{N} \subseteq\left(y_{n}\right)_{n=1}^{\infty}$ of $E$. Because $E$ is self-adjoint, from $\left\langle y_{n_{j}}, y_{n_{k}}\right\rangle=\left\langle y_{n_{j}}^{*}, y_{n_{k}}^{*}\right\rangle=\delta_{j k}$ it follows that $\left(y_{n_{j}}^{*}\right)_{j=1}^{N}$ is an orthonormal basis of $E$. Define self-adjoint operators $a_{j}=y_{n_{j}}+y_{n_{j}}^{*}$ and $a_{N+j}=i\left(y_{n_{j}}-y_{n_{j}}^{*}\right)$ for each $1 \leq j \leq N$ so that $E=\operatorname{Span}\left(a_{j}\right)_{j=1}^{2 N}$. We follow the Gram-Schmidt process and set $b_{1}=a_{1}$ and recursively define

$$
b_{k}=a_{k}-\sum_{j=1}^{k-1} \frac{\left\langle b_{j}, a_{k}\right\rangle}{\left\langle b_{j}, b_{j}\right\rangle} b_{j}
$$

to produce a sequence of $N$ many orthogonal operators which span $E$ (the remaining $N$ many operators produced by the Gram-Schmidt process become zero). Straight forward calculation reveals that $\left\langle a_{j}, a_{k}\right\rangle$ is real for every $1 \leq j, k \leq 2 N$, and hence $\left\langle b_{j}, a_{k}\right\rangle$ is real for every $1 \leq j, k \leq 2 N$. Each $b_{k}$ is thus self-adjoint as a real combination of self-adjoint operators, and the set $\left(b_{k}\right)_{k=1}^{N}$ can therefore be normalized to a set of self-adjoint orthonormal operators $\left(x_{j}\right)_{j=1}^{N}$ which span $E$. Replacing $y_{n}$ with $x_{n}$ in the original eigensystem decomposition for each eigenspace $E$, we have

$$
A=\sum_{n=1}^{\infty} \lambda_{n}\left|x_{n}\right\rangle\left\langle x_{n}\right|,
$$

as desired.

Lemma 3 Let $\mathcal{H}$ be a Hilbert space and $\widetilde{L}$ be a bounded linear operator on $\mathcal{S}_{2}(\mathcal{H})$ which has the form (22). Then $\widetilde{L}$ is conditionally completely positive if and only if for some (equivalently all) normalized vector $e \in \mathcal{H}$, the operator $\widetilde{L}_{\otimes, e}$ defined on the Hilbert space $\mathcal{S}_{2}(\mathcal{H}) \otimes \mathcal{H}$, by Equation 24, is positive.

Proof First note that $I+A$ is conditionally completely positive if and only if $A$ is (as is easily verified), so for simplicity we may assume instead that $\widetilde{L}=\sum_{n=1}^{\infty} \lambda_{n}\left|a_{n}\right\rangle\left\langle b_{n}\right|$.

We will start with the forward direction and suppose $\widetilde{L}$ is conditionally completely positive. Let $e \in \mathcal{H}$ with $\|e\|=1$. Since $W=\left\{\sum_{i=1}^{k} y_{i} \otimes h_{i}^{\prime}\right.$ : $\left.y_{i} \in \mathcal{S}_{2}(\mathcal{H}), h_{i}^{\prime} \in \mathcal{H}\right\}$ is dense in $\mathcal{S}_{2}(\mathcal{H}) \otimes \mathcal{H}$, in order to verify that $\widetilde{L}_{\otimes, e} \geq 0$ it is enough to consider an element $w=\sum_{i=1}^{k} y_{i} \otimes h_{i}^{\prime} \in W$ and verify that $\left\langle w, \widetilde{L}_{\otimes, e} w\right\rangle_{\otimes} \geq 0$, where $\langle\cdot, \cdot\rangle_{\otimes}$ will denote the inner product of $\mathcal{S}_{2}(\mathcal{H}) \otimes \mathcal{H}$. 
(The reason that we chose $h_{i}^{\prime}$ to denote a generic element of $\mathcal{H}$ is because we have used $h_{n}$ to denote the orthonormal eigenvectors of $\rho$ in the statement of Theorem (4). We will denote the inner product of $\mathcal{H}$ by $\langle\cdot, \cdot\rangle_{\mathcal{H}}$. Fix $w=$ $\sum_{i=1}^{k} y_{i} \otimes h_{i}^{\prime} \in W$ and let $v=-\sum_{i=1}^{k} y_{i}\left(h_{i}^{\prime}\right)$. Define $y_{k+1}=|v\rangle\langle e|$ and $h_{k+1}^{\prime}=$ $e$. Then $\sum_{i=1}^{k+1} y_{i}\left(h_{i}^{\prime}\right)=0$ and, since $\widetilde{L}$ is conditionally completely positive, we have that

$$
\begin{aligned}
0 & \leq \sum_{i, j=1}^{k+1}\left\langle h_{i}^{\prime}, \widetilde{L}\left(y_{i}^{*} y_{j}\right) h_{j}^{\prime}\right\rangle_{\mathcal{H}} \\
& =\sum_{i, j=1}^{k+1} \sum_{n=1}^{\infty} \lambda_{n} \operatorname{Tr}\left(y_{i}^{*} y_{j} b_{n}\right)\left\langle h_{i}^{\prime}, a_{n}\left(h_{j}^{\prime}\right)\right\rangle_{\mathcal{H}} \\
& =\sum_{i, j=1}^{k+1} \sum_{n=1}^{\infty} \lambda_{n}\left\langle y_{i} \otimes h_{i}^{\prime}, y_{j} b_{n} \otimes a_{n}\left(h_{j}^{\prime}\right)\right\rangle_{\otimes} \\
& \left.=\sum_{i, j=1}^{k+1} \sum_{n=1}^{\infty}\left\langle y_{i} \otimes h_{i}^{\prime}, \lambda_{n} M_{b_{n}} \otimes a_{n}\left(y_{j} \otimes h_{j}^{\prime}\right)\right\rangle_{\otimes} \otimes\left(\sum_{j=1}^{k+1} y_{j} \otimes h_{j}^{\prime}\right)\right\rangle_{\otimes} . \\
& =\left\langle\sum_{i=1}^{k+1} y_{i} \otimes h_{i}^{\prime},\left(\sum_{n=1}^{\infty} \lambda_{n} M_{b_{n}} \otimes a_{n}\right)\right.
\end{aligned}
$$

Notice that

$$
\begin{aligned}
\sum_{i=1}^{k+1} y_{i} \otimes h_{i}^{\prime} & =\sum_{i=1}^{k} y_{i} \otimes h_{i}^{\prime}+y_{k+1} \otimes h_{k+1}^{\prime}=w-\sum_{i=1}^{k}\left|y_{i}\left(h_{i}^{\prime}\right)\right\rangle\langle e| \otimes e \\
& =w-T_{e}\left(\sum_{i=1}^{k} y_{i} \otimes h_{i}^{\prime}\right)=\left(I d-T_{e}\right)(w)
\end{aligned}
$$

where $I d$ denotes the identity operator on $\mathcal{S}_{2}(\mathcal{H}) \otimes \mathcal{H}$, which finishes the proof of the forward direction.

For the other direction, suppose that $\widetilde{L}_{\otimes, e} \geq 0$ for some $e \in \mathcal{H}$ with $\|e\|=1$. Let $k \in \mathbb{N}, y_{1}, \ldots, y_{k} \in \mathcal{S}_{2}(\mathcal{H})$ and $h_{1}^{\prime}, \ldots, h_{k}^{\prime} \in \mathcal{H}$ such that $\sum_{i=1}^{k} y_{i}\left(h_{i}^{\prime}\right)=0$. Let $w=\sum_{i=1}^{k} y_{i} \otimes h_{i}^{\prime} \in \mathcal{S}_{2}(\mathcal{H}) \otimes \mathcal{H}$. Then,

$$
\begin{aligned}
0 & \leq\left\langle w, \widetilde{L}_{\otimes, e}(w)\right\rangle_{\otimes} \\
& =\left\langle w,\left(I d-T_{e}\right)^{*}\left(\sum_{n \in N} \lambda_{n} M_{b_{n}} \otimes a_{n}\right)\left(I d-T_{e}\right)(w)\right\rangle_{\otimes} \\
& =\left\langle\left(I d-T_{e}\right) w,\left(\sum_{n \in N} \lambda_{n} M_{b_{n}} \otimes a_{n}\right)\left(I d-T_{e}\right)(w)\right\rangle_{\otimes} .
\end{aligned}
$$


Notice that

$$
T_{e}(w)=T_{e}\left(\sum_{i=1}^{k} y_{i} \otimes h_{i}^{\prime}\right)=\left|\sum_{i=1}^{k} y_{i}\left(h_{i}^{\prime}\right)\right\rangle\langle e|\otimes e=| 0\rangle\langle e| \otimes e=0 .
$$

Hence Inequality (25) gives

$$
\begin{aligned}
0 & \leq\left\langle I d(w),\left(\sum_{n \in N} \lambda_{n} M_{b_{n}} \otimes a_{n}\right) I d(w)\right\rangle_{\otimes} \\
& =\sum_{i, j=1}^{k} \sum_{n \in N}\left\langle y_{i} \otimes h_{i}^{\prime}, \lambda_{n} M_{b_{n}} \otimes a_{n}\left(y_{j} \otimes h_{j}^{\prime}\right)\right\rangle_{\otimes} \\
& =\sum_{i, j=1}^{k} \sum_{n \in N} \lambda_{n}\left\langle y_{i} \otimes h_{i}^{\prime}, y_{j} b_{n} \otimes a_{n}\left(h_{j}^{\prime}\right)\right\rangle_{\otimes} \\
& =\sum_{i, j=1}^{k} \sum_{n \in N} \lambda_{n} \operatorname{Tr}\left(y_{i}^{*} y_{j} b_{n}\right)\left\langle h_{i}^{\prime}, a_{n}\left(h_{j}^{\prime}\right)\right\rangle_{\mathcal{H}} \\
& =\sum_{i, j=1}^{k+1}\left\langle h_{i}, \tilde{L}\left(y_{i}^{*} y_{j}\right) h_{j}^{\prime}\right\rangle_{\mathcal{H}} .
\end{aligned}
$$

Therefore $\widetilde{L}$ is conditionally completely positive. This completes the proof.

The proof of Lemma 3 reveals the following:

Remark 11 Let $\mathcal{A}=\left\{\sum_{i=1}^{k} y_{i} \otimes h_{i}^{\prime} \in \mathcal{B}(\mathcal{H}) \otimes \mathcal{H}: \sum_{i=1}^{k} y_{i}\left(h_{i}^{\prime}\right)=0\right\}$. Then

- For every $w=\sum_{i=1}^{k} y_{i} \otimes h_{i}^{\prime} \in \mathcal{B}(\mathcal{H}) \otimes \mathcal{H}$ there exists $y_{k+1} \in \mathcal{B}(\mathcal{H})$ and $h_{k+1}^{\prime} \in \mathcal{H}$ such that $\sum_{i=1}^{k+1} y_{i} \otimes h_{i}^{\prime} \in \mathcal{A}$ and $\left(I d-T_{h_{k+1}^{\prime}}\right)(w)=\sum_{i=1}^{k+1} y_{i} \otimes h_{i}^{\prime}$.

- If a bounded operator $\widetilde{L}$ on $\mathcal{H}$ has form (22) then $\widetilde{L}$ is completely positive if and only if the operator $\sum_{n=1}^{\infty} \lambda_{n} M_{b_{n}} \otimes a_{n}: S_{2}(\mathcal{H}) \otimes \mathcal{H} \rightarrow S_{2}(\mathcal{H}) \otimes \mathcal{H}$ is positive.

- For every $e \in \mathcal{H}$ we have $\mathcal{A} \subseteq \operatorname{ker} T_{e}$.

We are now ready to present the

Proof (Proof of Theorem 4) Since $\widetilde{L}$ generates a strongly continuous semigroup of contractions, we have that $\lambda \in \rho(\widetilde{L})$ for all $\lambda>0$ by the Hille-Yosida Generation Theorem (e.g. Theorem 3.5 of [16]). Further, $D(\widetilde{L})$ is dense in $S_{2}(\mathcal{H})$ by Theorem 3.1 .16 of [6] and $\widetilde{L}$ is star-preserving by Corollary 1 , and so $K:=(\widetilde{L}-I)^{-1}$ is star-preserving by Lemma 1 as the inverse of a starpreserving map with dense domain. Because $\widetilde{L}$ has compact resolvent by assumption, we have that $K$ is furthermore compact. Thus, $K^{\dagger} K$ is compact, self-adjoint, and star-preserving, and so Lemma 2 implies

$$
K^{\dagger} K=\sum_{n=1}^{\infty} \sigma_{n}^{2}\left|v_{n}\right\rangle\left\langle v_{n}\right|
$$


where $\left\{\sigma_{n}^{2}\right\}_{n \in \mathbb{N}}$ are the nonzero eigenvalues of $K^{\dagger} K$ corresponding to the system $\left\{v_{n}\right\}_{n \in \mathbb{N}}$ of self-adjoint orthonormal eigenoperators. This notation is chosen so that, following Section 2.2 of [17, the singular value expansion of $K$ can be written

$$
K=\sum_{n=1}^{\infty} \sigma_{n}\left|u_{n}\right\rangle\left\langle v_{n}\right|,
$$

where $\left\{u_{n}\right\}_{n \in \mathbb{N}}$ are self-adjoint orthonormal eigenoperators of $K K^{\dagger}$ given by the relation $\sigma_{n} u_{n}:=K v_{n}$. By Theorem 2.8 of [17] we have that

$$
\widetilde{L}-I=K^{(-1)}=\sum_{n=1}^{\infty} \frac{1}{\sigma_{n}}\left|v_{n}\right\rangle\left\langle u_{n}\right|,
$$

and hence

$$
\widetilde{L}=I+\sum_{n=1}^{\infty} \frac{1}{\sigma_{n}}\left|v_{n}\right\rangle\left\langle u_{n}\right|,
$$

proving (22). Equation (23) follows from (22) and (17). Part (c) is valid since $L(I)=0$, hence $L_{\left(h_{n}\right)}(I)=0$, and hence

$$
I+\sum_{n=1}^{\infty} \lambda_{n}\left\langle h_{n}, \rho^{1 / 2}\right\rangle i_{\rho^{(-1)}}\left(a_{n}\right)=0 .
$$

Part (d) follows from Lemma 3 indeed, $\left(\widetilde{T}_{t}\right)_{t \geq 0}$ is a completely positive semigroup by Proposition 3 and so $\widetilde{L}$ is conditionally completely positive by Theorem 3

Remark 12 Let $\mathcal{H}$ be a finite dimensional Hilbert space, and let $\left(T_{t}\right)_{t \geq 0}$ be a QMS on $\mathcal{B}(\mathcal{H})$. Then $\left(T_{t}\right)_{t \geq 0}$ possesses an invariant faithful normal state 23 , Theorem 4.2] and so Theorem 4 applies. The sum in (23) is finite, and hence by linearity/conjugate linearity we can write

$$
\begin{aligned}
L_{\left(h_{n}\right)} & =I+\sum_{n} \lambda_{n}\left|i_{\rho^{(-1)}}\left(a_{n}^{+}-a_{n}^{-}\right)\right\rangle\left\langle i_{\rho}\left(b_{n}^{+}-b_{n}^{-}\right)\right| \\
& =I+\sum_{m} \lambda_{m}^{\prime}\left|i_{\rho^{(-1)}}\left(c_{m}\right)\right\rangle\left\langle i_{\rho}\left(d_{m}\right)\right|,
\end{aligned}
$$

where $c_{m} \in\left\{a_{n}^{+}, a_{n}^{-}: n\right\}, d_{m} \in\left\{b_{n}^{+}, b_{n}^{-}: n\right\}$, and $\lambda_{m}^{\prime} \in\left\{ \pm \lambda_{n}: n\right\}$. In particular, $c_{m}, d_{m} \geq 0$ and $\lambda_{m}^{\prime} \in \mathbb{R}$. Thus, for every $x \in \mathcal{B}(\mathcal{H})$,

$$
\begin{aligned}
\left|i_{\rho^{(-1)}}\left(c_{m}\right)\right\rangle\left\langle i_{\rho}\left(d_{m}\right)\right| x & =\left\langle i_{\rho}\left(d_{m}\right), x\right\rangle i_{\rho^{(-1)}}\left(c_{m}\right) \\
& =\left\langle\rho^{1 / 4} d_{m} \rho^{1 / 4}, x\right\rangle \rho^{-1 / 4} c_{m} \rho^{-1 / 4} \\
& =\rho^{-1 / 4} \sqrt{c_{m}} \operatorname{Tr}\left(\rho^{1 / 4} d_{m} \rho^{1 / 4} x\right) \sqrt{c_{m}} \rho^{-1 / 4} \\
& =\rho^{-1 / 4} \sqrt{c_{m}} \operatorname{Tr}\left(\sqrt{d_{m}} \rho^{1 / 4} x \rho^{1 / 4} \sqrt{d_{m}}\right) \sqrt{c_{m}} \rho^{-1 / 4} .
\end{aligned}
$$


Now, fix any orthonormal basis $\left(E_{k}\right)_{k}$ of $\mathcal{B}(\mathcal{H})$. Then for every $A \in \mathcal{B}(\mathcal{H})$ we have

$$
\operatorname{Tr}(A)=\sum_{k} E_{k} A E_{k}^{*}
$$

Thus,

$$
\operatorname{Tr}\left(\sqrt{d_{m}} \rho^{1 / 4} x \rho^{1 / 4} \sqrt{d_{m}}\right)=\sum_{k} E_{k} \sqrt{d_{m}} \rho^{1 / 4} x \rho^{1 / 4} \sqrt{d_{m}} E_{k}^{*},
$$

and so

$$
L_{\left(h_{n}\right)}(x)=x+\sum_{m, k} \lambda_{m}^{\prime} \rho^{-1 / 4} \sqrt{c_{m}} E_{k} \sqrt{d_{m}} \rho^{1 / 4} x \rho^{1 / 4} \sqrt{d_{m}} E_{k}^{*} \sqrt{c_{m}} \rho^{-1 / 4} .
$$

By defining $y_{\ell}=\rho^{-1 / 4} \sqrt{c_{m}} E_{k} \sqrt{d_{m}} \rho^{1 / 4}$ and $\lambda_{\ell}^{\prime}$ to be the corresponding $\lambda_{m}^{\prime}$, we obtain

$$
L_{\left(h_{n}\right)}(x)=x+\sum_{\ell} \lambda_{\ell}^{\prime} y_{\ell} x y_{\ell}^{*}
$$

Moreover, since $L_{\left(h_{n}\right)}(I)=L(I)=0$ we obtain as in the proof of Theorem 4 (c) that

$$
I+\sum_{\ell} \lambda_{\ell} y_{\ell} y_{\ell}^{*}=0
$$

Thus

$$
L_{\left(h_{n}\right)}(x)=\frac{1}{2} I x+\frac{1}{2} x I+\sum_{\ell} \lambda_{\ell}^{\prime} y_{\ell} x y_{\ell}^{*}=\sum_{\ell} \lambda_{\ell}^{\prime}\left(y_{\ell} x y_{\ell}^{*}-\frac{1}{2}\left\{y_{\ell} y_{\ell}^{*}, x\right\}\right)
$$

which resembles the standard GKSL form developed in [28] and [36, except with Hamiltonian part zero and without the demand that the jump operators $y_{\ell}$ are traceless. A comparison of such GKSL form to the standard GKSL form, including conversion between the two, is discussed in section 2.1 of [2].

\section{Conflict of interest}

The authors declare that they have no conflict of interest.

Acknowledgements We would like to thank Franco Fagnola. His contributions to the results of this work were vital from its conception to the final touches. Without his help the existence of this work would not be possible. We would also like to thank the referee for pointing our attention to a mistake in the original version of this article. 


\section{References}

1. Alazzawi, S., Baumgartner, B.: Generalized Kraus operators and generators of quantum dynamical semigroups. Rev. Math. Phys. 27(7), 1550016, 19 (2015)

2. Androulakis, G., Wiedemann, A.: GKSL generators and digraphs: computing invariant states. Journal of Physics A: Mathematical and Theoretical 52(30), 305201 (2019)

3. Androulakis, G., Ziemke, M.: Generators of quantum Markov semigroups. J. Math. Phys. 56(8), 083512, 16 (2015)

4. Attal, S.: Lecture 3: Operator semigroups. In: Lectures in Quantum Noise Theory Retrieved from http://math.univ-lyon1.fr/ attal/

5. Bhatt, S.J.: Stinespring representability and Kadison's Schwarz inequality in non-unital Banach star algebras and applications. Proc. Indian Acad. Sci. Math. Sci. 108(3), 283-303 (1998)

6. Bratteli, O., Robinson, D.W.: Operator algebras and quantum statistical mechanics. 1, second edn. Texts and Monographs in Physics. Springer-Verlag, New York (1987). $C^{*}-$ and $W^{*}$-algebras, symmetry groups, decomposition of states

7. Carbone, R., Fagnola, F.: Exponential convergence in $L^{2}$ of quantum markov semigroups on $\mathcal{B}(\mathcal{H})$. Mat. Zametki 68(4), 523-538 (2000)

8. Carbone, R., Sasso, E., Umanità, V.: Environment induced decoherence for Markovian evolutions. J. Math. Phys. 56(9), 092704, 22 (2015)

9. Chebotarev, A.M., Fagnola, F.: Sufficient conditions for conservativity of quantum dynamical semigroups. J. Funct. Anal. 118(1), 131-153 (1993)

10. Chebotarev, A.M., Fagnola, F.: Sufficient conditions for conservativity of minimal quantum dynamical semigroups. J. Funct. Anal. 153(2), 382-404 (1998)

11. Choi, M.D.: A Schwarz inequality for positive linear maps on $C^{*}$-algebras. Illinois J. Math. 18, 565-574 (1974)

12. Christensen, E., Evans, D.E.: Cohomology of operator algebras and quantum dynamical semigroups. J. London Math. Soc. (2) 20(2), 358-368 (1979)

13. Davies, E.B.: Quantum dynamical semigroups and the neutron diffusion equation. Rep. Mathematical Phys. 11(2), 169-188 (1977)

14. Davies, E.B.: Generators of dynamical semigroups. J. Funct. Anal. 34(3), 421-432 (1979)

15. Dunford, N., Schwartz, J.T.: Linear operators. Part II: Spectral theory. Self adjoint operators in Hilbert space. With the assistance of William G. Bade and Robert G. Bartle. Interscience Publishers John Wiley \& Sons New York-London (1963)

16. Engel, K.J., Nagel, R.: One-parameter semigroups for linear evolution equations, Graduate Texts in Mathematics, vol. 194. Springer-Verlag, New York (2000). With contributions by S. Brendle, M. Campiti, T. Hahn, G. Metafune, G. Nickel, D. Pallara, C. Perazzoli, A. Rhandi, S. Romanelli and R. Schnaubelt

17. Engl, H.W., Hanke, M., Neubauer, A.: Regularization of inverse problems, Mathematics and its Applications, vol. 375. Kluwer Academic Publishers Group, Dordrecht (1996)

18. Evans, D.E.: Conditionally completely positive maps on operator algebras. Quart. J. Math. Oxford Ser. (2) 28(111), 271-283 (1977)

19. Evans, D.E., Lewis, J.T.: Dilations of dynamical semi-groups. Comm. Math. Phys. 50(3), 219-227 (1976)

20. Fagnola, F.: Quantum Markov semigroups and quantum flows. Proyecciones 18(3), 144 (1999)

21. Fagnola, F., Rebolledo, R.: The approach to equilibrium of a class of quantum dynamical semigroups. Infin. Dimens. Anal. Quantum Probab. Relat. Top. 1(4), 561-572 (1998)

22. Fagnola, F., Rebolledo, R.: On the existence of stationary states for quantum dynamical semigroups. J. Math. Phys. 42(3), 1296-1308 (2001)

23. Fagnola, F., Rebolledo, R.: Some results on invariant states for quantum markov semigroups. In: G. Da Prato, L. Tubaro (eds.) Stochastic Partial Differential Equations and Applications, pp. 197-208. CRC Press, Boca Raton (2002)

24. Fagnola, F., Rebolledo, R.: Quantum Markov semigroups and their stationary states. In: Stochastic analysis and mathematical physics II, Trends Math., pp. 77-128. Birkhäuser, Basel (2003) 
25. Fagnola, F., Rebolledo, R.: Algebraic conditions for convergence of a quantum Markov semigroup to a steady state. Infin. Dimens. Anal. Quantum Probab. Relat. Top. 11(3), 467-474 (2008)

26. Frigerio, A.: Quantum dynamical semigroups and approach to equilibrium. Lett. Math. Phys. 2(2), 79-87 (1977/78)

27. Frigerio, A.: Stationary states of quantum dynamical semigroups. Comm. Math. Phys. 63(3), 269-276 (1978)

28. Gorini, V., Kossakowski, A., Sudarshan, E.C.G.: Completely positive dynamical semigroups of $N$-level systems. J. Mathematical Phys. 17(5), 821-825 (1976)

29. Holevo, A.S.: On conservativity of covariant dynamical semigroups. In: Proceedings of the XXV Symposium on Mathematical Physics (Toruń, 1992), vol. 33, pp. 95-110 (1993)

30. Holevo, A.S.: On the structure of covariant dynamical semigroups. J. Funct. Anal. 131(2), 255-278 (1995)

31. Holevo, A.S.: Statistical structure of quantum theory, Lecture Notes in Physics. Monographs, vol. 67. Springer-Verlag, Berlin (2001)

32. Kadison, R.V.: A generalized Schwarz inequality and algebraic invariants for operator algebras. Ann. of Math. (2) 56, 494-503 (1952)

33. Kadison, R.V., Ringrose, J.R.: Fundamentals of the theory of operator algebras. Vol. I, Graduate Studies in Mathematics, vol. 15. American Mathematical Society, Providence, RI (1997). Elementary theory, Reprint of the 1983 original

34. Kossakowski, A.: On quantum statistical mechanics of non-Hamiltonian systems. Rep. Mathematical Phys. 3(4), 247-274 (1972)

35. Kossakowski, A., Frigerio, A., Gorini, V., Verri, M.: Quantum detailed balance and KMS condition. Comm. Math. Phys. 57(2), 97-110 (1977)

36. Lindblad, G.: On the generators of quantum dynamical semigroups. Comm. Math. Phys. 48(2), 119-130 (1976)

37. Ohya, M., Petz, D.: Quantum entropy and its use. Texts and Monographs in Physics. Springer-Verlag, Berlin (1993)

38. Siemon, I., Holevo, A.S., Werner, R.F.: Unbounded generators of dynamical semigroups. Open Syst. Inf. Dyn. 24(4), 1740015, 24 (2017)

39. Sinha, K.B.: Quantum dynamical semigroups. In: Mathematical results in quantum mechanics, Oper. Theory Adv. Appl., vol. 70, pp. 161-169. Birkhäuser, Basel (1994)

40. Størmer, E.: Positive linear maps of operator algebras. Acta Math. 110, 233-278 (1963)

41. Størmer, E.: Positive linear maps of operator algebras. Springer Monographs in Mathematics. Springer, Heidelberg (2013)

42. Umanità, V.: Classification and decomposition of quantum Markov semigroups. Probab. Theory Related Fields 134(4), 603-623 (2006)

43. Wiedemann, A.: On the generators of quantum dynamical semigroups. (doctoral dissertation) (2019). Retrieved from https://scholarcommons.sc.edu/etd/5231 